\title{
Rewriting systems and biautomatic structures for Chinese, hypoplactic, and sylvester monoids
}

\author{
Alan J. Cain, Robert D. Gray, António Malheiro
}

[AJC] Centro de Matemática e Aplicações, Faculdade de Ciências e Tecnologia, Universidade Nova de Lisboa, 2829-516 Caparica, Portugal

Email: a.cain@fct.unl.pt

Web page: www.fc.up.pt/pessoas/ajcain/

[RDG] School of Mathematics, University of East Anglia, Norwich NR4 7TJ, United Kingdom

Email: Robert.D.Gray@uea.ac.uk

[AM] Departamento de Matemática, Faculdade de Ciências e Tecnologia, Universidade Nova de Lisboa, 2829-516 Caparica, Portugal and Centro de Álgebra da Universidade de Lisboa, Av. Prof. Gama Pinto 2, 1649-003 Lisboa, Portugal

Email: ajm@fct.unl.pt

Dedicated to Stuart W. Margolis on the occasion of his 6oth birthday

\begin{abstract}
This paper studies complete rewriting systems and biautomaticity for three interesting classes of finite-rank homogeneous monoids: Chinese monoids, hypoplactic monoids, and sylvester monoids. For Chinese monoids, we first give new presentations via finite complete rewriting systems, using more lucid constructions and proofs than those given independently by Chen $\mathcal{E}$ Qui and Güzel Karpuz; we then construct biautomatic structures. For hypoplactic monoids, we construct finite complete rewriting systems and biautomatic structures. For sylvester monoids, which are not finitely presented, we prove that the standard presentation is an infinite complete rewriting system, and construct biautomatic structures. Consequently, the monoid algebras corresponding to monoids of these classes are automaton algebras in the sense of Ufnarovskij.

Acknowledgements: During the research that led to this paper, the first author was supported by an Investigador FCT fellowship (IF/01622/2013/CP1161/CTooo1). This work was developed within the research activities of the Centro de Álgebra da Universidade de Lisboa, FCT project PEsT-OE/MAT/UIo143/2014, and of the Departamento de Matemática da Faculdade de Ciências e Tecnologia da Universidade Nova de Lisboa.
\end{abstract}


The aim of this paper is to study whether certain homogeneous monoids admit presentations via finite complete rewriting systems or are biautomatic. The focus is on Chinese monoids, hypoplactic monoids, and sylvester monoids of finite rank. All three classes of monoids are related to Plactic monoids. In a previous paper [CGM15], we answered a question of Zelmanov by constructing biautomatic structures and presentations via finite complete rewriting systems for Plactic monoids of finite rank. The present paper is partly motivated by that earlier work, but the techniques we use here are original. As we discuss below, our results also have consequences for the study of the corresponding monoid algebras.

The Chinese monoid was introduced by Duchamp \& Krob [DK94], as one of the (few) multi-homogeneous monoids with the same multihomogeneous growth as the Plactic monoid. Cassaigne et al. [CEK $\left.{ }^{+} \mathrm{OI}\right]$ made the first fundamental study of the Chinese monoid, and the Chinese algebra has also been studied [CO13, JOo6, JO11]. The result that finite-rank Chinese monoids are presented by finite complete rewriting systems was obtained in the context of Gröbner-Shirshov bases by Chen \& Qiu [CQ08], and later in the context of rewriting systems by Güzel Karpuz [GKIo]. In both approaches the standard presentation of the Chinese monoid is the starting point. Chen $\mathcal{E}$ Qiu apply the Shirshov algorithm; Güzel Karpuz applies the Knuth-Bendix completion procedure. These procedures parallel each other [Heyoo] and consist of adding rewriting rules which arise from the analysis of all possible overlaps. In both papers, proving confluence of the resulting rewriting system by checking the 37 possible critical pairs is left as an exercise for the interested reader. In $\S 3.2$, we use different generating sets to construct presentations via a finite complete rewriting systems for Chinese monoids: we think these rewriting systems are easier to understand and the proofs more elegant. We then proceed to prove that finite-rank Chinese monoids are biautomatic in $\S$ 3.5, exhibiting en route a left-handed analogue of the algorithm of Cassaigne et al. $\left[\mathrm{CEK}^{+} \mathrm{OI}, \S 2.2\right]$ for right-multiplying an element of the Chinese monoid by a generator.

The hypoplactic algebra was introduced by Krob \& Thibon [KT97] as a quotient of the Plactic algebra. The fundamental study of the underlying hypoplactic monoid, which is a quotient of the Plactic monoid, is due to Novelli [Novoo]. In $\S 4.2$, we give a neat construction and proof that finite-rank hypoplactic monoids admit presentations via finite complete rewriting systems. In $\S 4.3$, we prove that hypoplactic monoids of finite rank are biautomatic.

The sylvester monoid was defined by Hivert, Novelli $\mathcal{E}$ Thibon [HNTo5] as an analogue of the plactic monoid where Schensted's algorithm for insertion into Young tableaux (see [Loto2, ch. 5]) is replaced by insertion into a binary search tree; from the sylvester monoid, they then recover the Hopf algebra of planar binary trees defined by Loday $\mathcal{E}$ Ronco [LR98]. Finite-rank sylvester monoids are not finitely presented, but in $\S 5.1$, we show that the standard presentations for finite-rank sylvester monoids form (infinite) complete rewriting systems. In $\S 5.2$, we proceed to show that sylvester monoids of finite rank are biautomatic.

The existence of finite complete rewriting systems for the Chinese and hypoplactic monoids immediately implies the existence of finite GröbnerShirshov bases for the Chinese and hypoplactic algebras [Heyoo]. From the biautomaticity of the Chinese, hypoplactic, and sylvester monoids, we imme- 
diately recover the solvability of the word problem in quadratic time [CRRTo1, Corollary 3.7]. Furthermore, the biautomaticity of these monoids implies that each admits a regular cross-section [CRRTo1, Corollary 5.6], which in turn implies that the corresponding monoid algebras are automaton algebras in the sense of Ufarnovskij [Ufn95].

In related work [CGM], we give examples of homogeneous and multihomogeneous monoids that do not admit finite complete rewriting systems or biautomatic structures, and indeed we show that the two notions are independent within the classes of homogeneous and multihomogeneous monoids. Thus the results in this paper are not simply consequences of more general results for homogeneous or multi-homogeneous monoids.

Since Chinese, hypolactic, and sylvester monoids are biautomatic, they have decidable conjugacy problem. (To be precise, one can decide the oconjugacy relation, define by Otto [Ott84], using reasoning similar to the group case $\left[\mathrm{ECH}^{+}\right.$92, Theorem 2.5.7].) The algorithm for biautomatic monoids is exponential-time in general. An interesting open question is whether one can improve this exponential bound for homogeneous biautomatic monoids. (However, there are (non-biautomatic) homogeneous and even multihomogeneous monoids in which conjugacy is undecidable [CM, Theorem 4.1].)

\section{PRELIMINARIES}

\subsection{Words and presentations}

We denote the empty word (over any alphabet) by $\varepsilon$. For an alphabet $A$, we denote by $A^{*}$ the set of all words over $A$. When $A$ is a generating set for a monoid $M$, every element of $A^{*}$ can be interpreted either as a word or as an element of $M$. For words $u, v \in A^{*}$, we write $u=v$ to indicate that $u$ and $v$ are equal as words and $u={ }_{M} v$ to denote that $u$ and $v$ represent the same element of the monoid $M$. The length of $u \in A^{*}$ is denoted $|u|$, and, for any $a \in A$, the number of symbols $a$ in $u$ is denoted $|u|_{a}$.

For any relation $\mathcal{R}$ on $A^{*}$, the presentation $\langle A \mid \mathcal{R}\rangle$ defines [any monoid isomorphic to] $A^{*} / \mathcal{R}^{\#}$, where $\mathcal{R}^{\#}$ denotes the congruence generated by $\mathcal{R}$. The presentation $\langle A \mid \mathcal{R}\rangle$ is homogeneous (respectively, multi-homogeneous) if for every $(u, v) \in \mathcal{R}$ and $a \in A$, we have $|u|=|v|$ (respectively, $|u|_{a}=|v|_{a}$ ). That is, in a homogeneous presentation, defining relations preserve length; in a multi-homogenous presentation, defining relations preserve the numbers of each symbol. A monoid is homogeneous (respectively, multi-homogeneous) if it admits a homogeneous (respectively, multi-homogeneous) presentation.

Any total order $\leqslant$ on an alphabet $A$ induces a total order $\leqslant$ lex on $A^{*}$, where $w \leqslant_{\text {lex }} w^{\prime}$ if and only if either $w$ is proper prefix of $w^{\prime}$ or if $w=$ paq, $w^{\prime}=p b r$ and $a \leqslant b$ for some $p, q, r \in A^{*}$, and $a, b \in A$. The order $\leqslant_{\text {lex }}$ is the lexicographic order induced by $\leqslant$. Notice that $\leqslant_{\text {lex }}$ is not a well-order, but that it is left compatible with concatenation.

\subsection{String rewriting systems}

A string rewriting system, or simply a rewriting system, is a pair $(A, \mathcal{R})$, where $A$ is a finite alphabet and $\mathcal{R}$ is a set of pairs $(\ell, r)$, usually written $\ell \rightarrow r$, known as rewriting rules or simply rules, drawn from $A^{*} \times A^{*}$. The single reduction relation $\rightarrow_{\mathcal{R}}$ is defined as follows: $u \rightarrow_{\mathcal{R}} v$ (where $u, v \in A^{*}$ ) if there 
exists a rewriting rule $(\ell, r) \in \mathcal{R}$ and words $x, y \in A^{*}$ such that $u=x \ell y$ and $v=x r y$. That is, $u \rightarrow_{\mathcal{R}} v$ if one can obtain $v$ from $u$ by substituting the word $r$ for a subword $\ell$ of $u$, where $\ell \rightarrow r$ is a rewriting rule. The reduction relation $\rightarrow_{\mathcal{R}}^{*}$ is the reflexive and transitive closure of $\rightarrow_{\mathcal{R}}$. The process of replacing a subword $\ell$ by a word $r$, where $\ell \rightarrow r$ is a rule, is called reduction by application of the rule $\ell \rightarrow r$; the iteration of this process is also called reduction. A word $w \in A^{*}$ is reducible if it contains a subword $\ell$ that forms the left-hand side of a rewriting rule in $\mathcal{R}$; it is otherwise called irreducible.

The rewriting system $(A, \mathcal{R})$ is finite if both $A$ and $\mathcal{R}$ are finite. The rewriting system $(A, \mathcal{R})$ is noetherian if there is no infinite sequence $u_{1}, u_{2}, \ldots \in A^{*}$ such that $u_{i} \rightarrow_{\mathcal{R}} u_{i+1}$ for all $i \in \mathbb{N}$. That is, $(A, \mathcal{R})$ is noetherian if any process of reduction must eventually terminate with an irreducible word. The rewriting system $(A, \mathcal{R})$ is confluent if, for any words $u, u^{\prime}, u^{\prime \prime} \in A^{*}$ with $u \rightarrow_{\mathcal{R}}^{*} u^{\prime}$ and $u \rightarrow \rightarrow_{\mathcal{R}}^{*} u^{\prime \prime}$, there exists a word $v \in A^{*}$ such that $u^{\prime} \rightarrow_{\mathcal{R}}^{*} v$ and $u^{\prime \prime} \rightarrow_{\mathcal{R}}^{*} v$. A rewriting system that is both confluent and noetherian is complete. If $(A, \mathcal{R})$ is a complete rewriting system, then for every word $u$ there is a unique irreducible word $w$ such that $u \rightarrow_{\mathcal{R}}^{*} w$; this word is called the normal form of u.

The rewriting system $(A, \mathcal{R})$ is globally finite if, for each $w \in A^{*}$, there are only finitely many words $w^{\prime}$ such that $w \rightarrow_{\mathcal{R}}^{*} w^{\prime}$. It is acyclic if there is no word $w$ such that $w \rightarrow_{\mathcal{R}}^{+} w$. An acyclic globally finite rewriting system is noetherian [BN98, Lemma 2.2.5].

The Thue congruence $\leftrightarrow_{\mathcal{R}}^{*}$ is the equivalence relation generated by $\rightarrow_{\mathcal{R}}$. The elements of the monoid presented by $\langle A \mid \mathcal{R}\rangle$ are the $\leftrightarrow_{\mathcal{R}}^{*}$-equivalence classes. If $(A, \mathcal{R})$ is complete, then the language of normal form words forms a crosssection of the monoid: that is, each element of the monoid presented by $\langle A \mid \mathcal{R}\rangle$ has a unique normal form representive.

\subsection{Automaticity and biautomaticity}

This subsection contains the definitions and basic results from the theory of automatic and biautomatic monoids needed hereafter. For further information on automatic semigroups, see [CRRTo1]. We assume familiarity with basic notions of automata and regular languages (see, for example, [HU79]) and transducers and rational relations (see, for example, [Ber79]).

Definition 2.1. Let $A$ be an alphabet and let $\$$ be a new symbol not in $A$. Define the mapping $\delta_{R}: A^{*} \times A^{*} \rightarrow((A \cup\{\$\}) \times(A \cup\{\$\}))^{*}$ by

$$
\left(u_{1} \cdots u_{m}, v_{1} \cdots v_{n}\right) \mapsto \begin{cases}\left(u_{1}, v_{1}\right) \cdots\left(u_{m}, v_{n}\right) & \text { if } m=n \\ \left(u_{1}, v_{1}\right) \cdots\left(u_{n}, v_{n}\right)\left(u_{n+1}, \$\right) \cdots\left(u_{m}, \$\right) & \text { if } m>n \\ \left(u_{1}, v_{1}\right) \cdots\left(u_{m}, v_{m}\right)\left(\$, v_{m+1}\right) \cdots\left(\$, v_{n}\right) & \text { if } m<n\end{cases}
$$

and the mapping $\delta_{L}: A^{*} \times A^{*} \rightarrow((A \cup\{\$\}) \times(A \cup\{\$\}))^{*}$ by

$$
\left(u_{1} \cdots u_{m}, v_{1} \cdots v_{n}\right) \mapsto \begin{cases}\left(u_{1}, v_{1}\right) \cdots\left(u_{m}, v_{n}\right) & \text { if } m=n \\ \left(u_{1}, \$\right) \cdots\left(u_{m-n}, \$\right)\left(u_{m-n+1}, v_{1}\right) \cdots\left(u_{m}, v_{n}\right) & \text { if } m>n \\ \left(\$, v_{1}\right) \cdots\left(\$, v_{n-m}\right)\left(u_{1}, v_{n-m+1}\right) \cdots\left(u_{m}, v_{n}\right) & \text { if } m<n\end{cases}
$$

where $u_{i}, v_{i} \in A$.

Definition 2.2. Let $M$ be a monoid. Let $A$ be a finite alphabet representing a set of generators for $M$ and let $L \subseteq A^{*}$ be a regular language such that every 
element of $M$ has at least one representative in L. For each $a \in A \cup\{\varepsilon\}$, define the relations

$$
\begin{aligned}
& \mathrm{L}_{\mathrm{a}}=\left\{(\mathrm{u}, v): \mathrm{u}, v \in \mathrm{L}, \mathrm{ua}={ }_{M} v\right\} \\
& { }_{\mathrm{a}} \mathrm{L}=\left\{(\mathrm{u}, v): \mathrm{u}, v \in \mathrm{L}, \mathrm{au}={ }_{\mathrm{M}} v\right\} .
\end{aligned}
$$

The pair $(A, L)$ is an automatic structure for $M$ if $L_{a} \delta_{R}$ is a regular languages over $(A \cup\{\$\}) \times(A \cup\{\$\})$ for all $a \in A \cup\{\varepsilon\}$. A monoid $M$ is automatic if it admits an automatic structure with respect to some generating set.

The pair $(A, L)$ is a biautomatic structure for $M$ if $L_{a} \delta_{R},{ }_{a} L \delta_{R}, L_{a} \delta_{L}$, and ${ }_{a} L \delta_{L}$ are regular languages over $(A \cup\{\$\}) \times(A \cup\{\$\})$ for all $a \in A \cup\{\varepsilon\}$. A monoid $M$ is biautomatic if it admits a biautomatic structure with respect to some generating set. [Note that biautomaticity implies automaticity.]

Unlike the situation for groups, biautomaticity for monoids and semigroups, like automaticity, is dependent on the choice of generating set [CRRToI, Example 4.5]. However, for monoids, biautomaticity and automaticity are independent of the choice of semigroup generating sets [DRR99, Theorem 1.1].

Hoffmann $\mathcal{E}$ Thomas have made a careful study of biautomaticity for semigroups [HTo5]. They distinguish four notions of biautomaticity for semigroups:

- right-biautomaticity, where $\mathrm{L}_{\mathrm{a}} \delta_{\mathrm{R}}$ and ${ }_{\mathrm{a}} \mathrm{L} \delta_{\mathrm{R}}$ are regular languages;

- left-biautomaticity, where $\mathrm{L}_{\mathrm{a}} \delta_{\mathrm{L}}$ and ${ }_{\mathrm{a}} \mathrm{L} \delta_{\mathrm{L}}$ are regular languages;

- same-biautomaticity, where $\mathrm{L}_{\mathrm{a}} \delta_{\mathrm{R}}$ and ${ }_{\mathrm{a}} \mathrm{L} \delta_{\mathrm{L}}$ are regular languages;

- cross-biautomaticity, where ${ }_{a} \mathrm{~L} \delta_{\mathrm{R}}$ and $\mathrm{L}_{\mathrm{a}} \delta_{\mathrm{L}}$ are regular languages.

These notions are all equivalent for groups and more generally for cancellative semigroups [HT05, Theorem 1] but distinct for semigroups [HTo5, Remark 1 $\mathcal{E} \S$ 4]. In the sense used in this paper, 'biautomaticity' implies all four notions of biautomaticity above.

\subsection{Rational relations}

In proving that $R \delta_{R}$ or $R \delta_{L}$ is regular, where $R$ is a relation on $A^{*}$, a useful strategy is to prove that $R$ is a rational relation (that is, a relation recognized by a finite transducer [Ber79, Theorem 6.1]) and then apply the following result, which is a combination of [FS93, Corollary 2.5] and [HTo5, Proposition 4]:

Proposition 2.3. If $R \subseteq A^{*} \times A^{*}$ is rational relation and there is a constant $k$ such that || $\mathrm{u}|-| v|| \leqslant k$ for all $(\mathrm{u}, v) \in \mathrm{R}$, then $\mathrm{R} \delta_{\mathrm{R}}$ and $\mathrm{R} \delta_{\mathrm{L}}$ are regular.

REMARK 2.4. When constructing transducers to recognize particular relations, we will make use of certain strategies.

One strategy will be to consider a transducer reading elements of a relation $\mathrm{R}$ from right to left, instead of (as usual) left to right. In effect, such a transducer recognizes the reverse of $R$, which is the relation

$$
\mathrm{R}^{\mathrm{rev}}=\left\{\left(\mathrm{u}^{\mathrm{rev}}, v^{\mathrm{rev}}\right):(\mathrm{u}, v) \in \mathrm{R}\right\},
$$

where $u^{\text {rev }}$ and $v^{\text {rev }}$ are the reverses of the words $u$ and $v$ respectively. Since the class of rational relations is closed under reversal [Ber79, p.65-66], constructing such a (right-to-left) transducer suffices to show that $R$ is a rational relation. 
Another important strategy will be for the transducer to non-deterministically guess some symbol yet to be read. More exactly, the transducer will non-deterministically select a symbol and store it in its state. When it later reads the relevant symbol, it checks it against the stored guessed symbol. If the guess was correct, the transducer continues. If the guess was wrong, the transducer enters a failure state. Similarly, the transducer can non-deterministically guess that it has reached the end of its input and enter an accept state. If it subsequently reads another symbol, it knows that its guess was wrong, and it enters a failure state.

\section{CHINESE MONOID}

\subsection{Staircases}

Let $n \in \mathbb{N}$. Let $A$ be the finite ordered alphabet $\{1<2<\ldots<n\}$. Let $\mathcal{R}$ be the set of defining relations

$$
\begin{aligned}
& \{(z y x, z x y), \\
& \quad(z x y, y z x): x \leqslant y \leqslant z\} .
\end{aligned}
$$

Then the Chinese monoid of rank $n$, denoted $C_{n}$, is presented by $\langle A \mid \mathcal{R}\rangle$.

Cassaigne et al. [CEK ${ }^{+} \mathrm{OI}, \S 2$ ] give a set of normal forms for elements of the Chinese monoid. They point out that every element has a unique representative of the form

$$
\ell^{(1)} \ell^{(2)} \ell^{(3)} \cdots \ell^{(n)}
$$

with

$$
\ell^{(k)}=(k 1)^{\sigma_{k 1}}(k 2)^{\sigma_{k 2}} \cdots(k(k-1))^{\sigma_{k(k-1)}} k^{\sigma_{k}},
$$

where the exponents $\sigma_{k j}$ and $\sigma_{k}$ lie in $\mathbb{N}^{0}$. (Notice that in $(3.4), k-1$ is a single symbol.) Cassaige et al. arrange the exponents $\sigma_{k j}$ and $\sigma_{k}$ in a Chinese staircase, which is an analogue of the planar representation of a tableau for the Plactic monoid (see [Loto2, ch. 5]). For example, in the case $n=4$ the $\sigma_{k i}$ and $\sigma_{k}$ are arranged as follows:

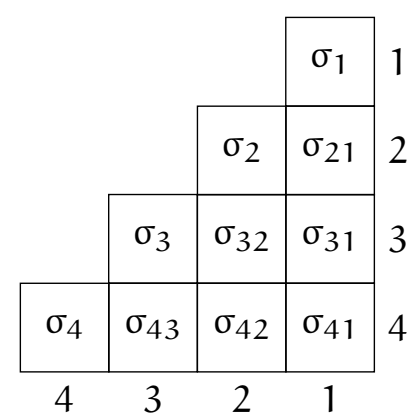

Notice that rows are indexed from top to bottom and columns from right to left. Because the $k$-th row of the staircase contains the exponents for the word $\ell^{(k)}$, each such word $\ell^{(k)}$ (see (3.4)) is called a Chinese row. A normal form word (3.3) is called a Chinese staircase word.

\subsection{Finite complete rewriting system}

As noted in the introduction, Chen $\mathcal{E}$ Qiu [CQo8] and Güzel Karpuz [GK10] independently constructed finite complete rewriting systems 
for finite-rank Chinese monoids. However, proving confluence of these systems relies on checking that all 37 possible critical pairs resolve. In this section we present an alternative finite complete rewriting system. By changing the generating set, we obtain a rewriting system whose language of irreducible words corresponds closely to the Chinese staircase words. Once we prove that the rewriting system is noetherian, we can deduce confluence quickly from the fact that Chinese staircase words form a set of unique representatives for the monoid.

Let

$$
\mathrm{D}=\left\{\mathrm{d}_{\alpha \beta}: \alpha, \beta \in A, \alpha>\beta\right\} \cup\left\{\mathrm{d}_{\alpha}: \alpha \in A\right\} .
$$

The idea is that symbols $d_{\alpha \beta}$ and $d_{\alpha}$ represent, respectively, the elements $\alpha \beta$ and $\alpha$ of $C_{n}$. Therefore the original generating set $A$ is essentially included in this new set, and so $D$ also generates $C_{n}$.

Consider a Chinese staircase word (3.3). By replacing each Chinese row $\ell^{(k)}$ (as in (3.4)) by

$$
d_{k 1}^{\sigma_{k 1}} d_{k 2}^{\sigma_{k 2}} \cdots d_{k(k-1)}^{\sigma_{k(k-1)}} d_{k}^{\sigma_{k}}
$$

we obtain a word over the generating set $D$ representing the same element of $\mathrm{C}_{n}$; we call such a word a D-Chinese staircase word. Notice that we can start from a D-Chinese staircase word and recover the original Chinese staircase word by simply reading off the subscripts of the symbols in D. Thus there is a one-to-one correspondence between Chinese staircase words and D-Chinese staircase words, and so the set of D-Chinese staircase words is also a language of unique normal forms for $C_{n}$.

Define a relation $\preceq$ on the alphabet $D$ as follows: for $d, d^{\prime} \in D$, we have $\mathrm{d} \preceq \mathrm{d}^{\prime}$ if and only if $\mathrm{dd}^{\prime}$ is a D-Chinese staircase word. As an immediate consequence of this definition, notice that $d \preceq d^{\prime}$ if and only if $d$ and $d^{\prime}$ can appear in that order, not necessarily as adjacent symbols, in a D-Chinese staircase word. Thus $\preceq$ is a total order.

Now define a set of rewriting rules $\mathcal{T}$ on $\mathrm{D}^{*}$ as follows:

$$
\mathcal{T}=\left\{\mathrm{dd}^{\prime} \rightarrow \mathrm{CS}_{\mathrm{D}}\left(\mathrm{dd}^{\prime}\right): \mathrm{d} \succ \mathrm{d}^{\prime}\right\},
$$

where $\mathrm{CS}_{\mathrm{D}}(w)$ denotes the unique D-Chinese staircase word that equals $\mathrm{dd}^{\prime}$ in $C_{n}$. It is obvious from the definition that $\mathcal{T}$ is finite and each rewriting rule in $\mathcal{T}$ holds in the Chinese monoid $C_{n}$.

The next lemma is immediate from the definition of $\mathcal{T}$.

LEMmA 3.1. The irreducible words of the rewriting system $(\mathrm{D}, \mathcal{T})$ are the D-Chinese staircase words.

As a consequence we deduce that the presentation $\langle\mathrm{D} \mid \mathcal{T}\rangle$ defines the Chinese monoid $C_{n}$. In the next lemma we show that $(D, \mathcal{T})$ is noetherian, and thus it will follow from the previous lemma that $(D, \mathcal{T})$ is complete. In order to show termination of $\mathcal{T}$ we will fully characterize the relation $\succ$ on $\mathrm{D}$.

Let $\alpha, \beta, \gamma, \delta \in A$. From the definition of $\preceq$ and the form of D-Chinese staircase words, we have:

$$
\begin{aligned}
\mathrm{d}_{\alpha} \succ \mathrm{d}_{\beta} & \Longleftrightarrow \alpha>\beta ; \\
\mathrm{d}_{\alpha \beta} \succ \mathrm{d}_{\gamma} & \Longleftrightarrow \alpha>\gamma ; \\
\mathrm{d}_{\alpha} \succ \mathrm{d}_{\beta \gamma} & \Longleftrightarrow \alpha \geqslant \beta ; \\
\mathrm{d}_{\alpha \beta} \succ \mathrm{d}_{\gamma \delta} & \Longleftrightarrow(\alpha>\gamma) \vee(\alpha=\gamma \wedge \beta>\delta) .
\end{aligned}
$$


Each of the left hand sides of a rewriting rule in $\mathcal{T}$ corresponds to one of the above cases. In the proof of the next lemma we will see the resulting Chinese staircase words for each of the left hand sides in $\mathcal{T}$.

LEMMA 3.2. The rewriting system $\mathcal{T}$ is noetherian.

Proof of 3.2. We will show that, for any $w, w^{\prime} \in \mathrm{D}^{*}$, if $w \rightarrow_{\mathcal{T}} w^{\prime}$ then $w \succ_{\text {lex }}$ $w^{\prime}$. Since $\succ_{\text {lex }}$ is left compatible with concatenation, it suffices to show that $\left(w_{1}, w_{2}\right) \in \mathcal{T}$ implies $w_{1} \succ_{\text {lex }} w_{2}$.

So let $\left(w_{1}, w_{2}\right) \in \mathcal{T}$. Depending on the form of $w_{1}$ we divide the proof into four cases:

1. $w_{1}=d_{\alpha} d_{\beta}$ with $d_{\alpha} \succ d_{\beta}$. Then $\alpha>\beta$. Therefore $C_{D}\left(d_{\alpha} d_{\beta}\right)=d_{\alpha \beta}$. We have $d_{\alpha} \succ d_{\alpha \beta}$ and so $d_{\alpha} d_{\beta} \succ_{\text {lex }} d_{\alpha \beta}$.

2. $w_{1}=d_{\alpha \beta} d_{\gamma}$ with $d_{\alpha \beta} \succ d_{\gamma}$. Then $\alpha>\gamma$ (and $\alpha>\beta$ by the definition of D). Now, consider two sub-cases:

(a) $\beta \geqslant \gamma$. Then

$$
\begin{aligned}
\alpha \beta \gamma & =c_{n} \alpha \gamma \beta & & \text { (by (3.1)) } \\
& =c_{n} \beta \alpha \gamma & & \text { (by (3.2)) }
\end{aligned}
$$

Therefore $C_{D}\left(d_{\alpha \beta} d_{\gamma}\right)=d_{\beta} d_{\alpha \gamma}$. Since $\alpha>\beta$, we have $d_{\alpha \beta} \succ d_{\beta}$ and so $d_{\alpha \beta} d_{\gamma} \succ_{\text {lex }} d_{\beta} d_{\alpha \gamma}$.

(b) $\gamma>\beta$. Then $\alpha \beta \gamma=C_{n} \gamma \alpha \beta$ by (3.2). Therefore $\operatorname{CS}_{D}\left(d_{\alpha \beta} d_{\gamma}\right)=d_{\gamma} d_{\alpha \beta}$. Since $\alpha>\gamma$, we have $d_{\alpha \beta} \succ d_{\gamma}$ and so $d_{\alpha \beta} d_{\gamma} \succ_{\text {lex }} d_{\gamma} d_{\alpha \beta}$.

3. If $w_{1}=d_{\alpha} d_{\beta \gamma}$ with $d_{\alpha} \succ d_{\beta \gamma}$. Then $\alpha \geqslant \beta$ (and $\beta>\gamma$ by the definition of D). We have

$$
\begin{aligned}
\alpha \beta \gamma & =c_{n} \alpha \gamma \beta & & \text { (by (3.1)) } \\
& =c_{n} \beta \alpha \gamma . & & \text { (by (3.2)) }
\end{aligned}
$$

Now consider two sub-cases:

(a) $\alpha=\beta$. Then $\operatorname{CS}_{D}\left(d_{\alpha} d_{\beta \gamma}\right)=d_{\alpha \gamma} d_{\beta}$. Since $\alpha>\gamma$, we have $d_{\alpha} \succ d_{\alpha \gamma}$ and so $d_{\alpha} d_{\beta \gamma} \succ_{\text {lex }} d_{\alpha \gamma} d_{\beta}$.

(b) $\alpha>\beta$. Then $\operatorname{CS}_{D}\left(d_{\alpha} d_{\beta \gamma}\right)=d_{\beta} d_{\alpha \gamma}$. Since $\alpha>\beta$, we have $d_{\alpha} \succ d_{\beta}$ and so $d_{\alpha} d_{\beta \gamma} \succ_{\text {lex }} d_{\beta} d_{\alpha \gamma}$.

4. If $w_{1}=d_{\alpha \beta} d_{\gamma \delta}$, with $d_{\alpha \beta} \succ d_{\gamma \delta}$. Then either $\alpha>\gamma$, or $\alpha=\gamma$ and $\beta>\delta$. (In both sub-cases, $\alpha>\beta$ and $\gamma>\delta$ by the definition of D.) Consider these sub-cases separately:

(a) $\alpha=\gamma$ and $\beta>\delta$. Then $\alpha=\gamma>\beta>\delta$, so

$$
\begin{aligned}
\alpha \beta \gamma \delta & =\alpha \beta \alpha \delta & & \\
& =c_{n} \alpha \alpha \beta \delta & & \text { (by (3.1) applied to } \alpha \beta \alpha) \\
& ={ }_{c_{n}} \alpha \alpha \delta \beta & & \text { (by (3.1) applied to } \alpha \beta \delta) \\
& ={ }_{c_{n}} \alpha \delta \alpha \beta & & \text { (by (3.1) applied to } \alpha \alpha \delta) \\
& =\gamma \delta \alpha \beta . & &
\end{aligned}
$$

Therefore $\operatorname{CS}_{D}\left(d_{\alpha \beta} d_{\gamma \delta}\right)=d_{\gamma \delta} d_{\alpha \beta}$. Since $\alpha=\gamma$ and $\beta>\delta$, we have $\mathrm{d}_{\alpha \beta} \succ \mathrm{d}_{\gamma \delta}$ and so $\mathrm{d}_{\alpha \beta} \mathrm{d}_{\gamma \delta} \succ_{\text {lex }} \mathrm{d}_{\gamma \delta} \mathrm{d}_{\alpha \beta}$.

(b) $\alpha>\gamma$. Now consider two sub-sub-cases separately: 
i. $\beta \geqslant \gamma$. Then $\alpha>\beta \geqslant \gamma>\delta$ and so

$$
\begin{aligned}
\alpha \beta \gamma \delta & =c_{n} \alpha \gamma \beta \delta & & \text { (by (3.1) applied to } \alpha \beta \gamma) \\
& =c_{n} \beta \alpha \gamma \delta & & \text { (by (3.2) applied to } \alpha \gamma \beta) \\
& =c_{n} \beta \alpha \delta \gamma & & \text { (by (3.1) applied to } \alpha \gamma \delta) \\
& =c_{n} \beta \gamma \alpha \delta . & & \text { (by (3.2) applied to } \alpha \delta \gamma)
\end{aligned}
$$

Consider two sub-sub-sub-cases separately:

A. $\beta=\gamma$. Then $\operatorname{CS}_{D}\left(d_{\alpha \beta} d_{\gamma \delta}\right)=d_{\beta}^{2} d_{\alpha \delta}$. Since $\alpha>\beta$, we have $d_{\alpha \beta} \succ d_{\beta}$ and so $d_{\alpha \beta} d_{\gamma \delta} \succ_{\text {lex }} d_{\beta}^{2} d_{\alpha \delta}$.

B. $\beta>\gamma$. Then $\operatorname{CS}_{D}\left(d_{\alpha \beta} d_{\gamma \delta}\right)=d_{\beta \gamma} d_{\alpha \delta}$. Since $\alpha>\beta$, we have $\mathrm{d}_{\alpha \beta} \succ \mathrm{d}_{\beta \gamma}$ and so $\mathrm{d}_{\alpha \beta} \mathrm{d}_{\gamma \delta} \succ_{\text {lex }} \mathrm{d}_{\beta \gamma} \mathrm{d}_{\alpha \delta}$.

ii. $\gamma>\beta$. Then $\alpha>\gamma>\beta, \delta$ and so $\alpha \beta \gamma \delta={ }_{c_{n}} \gamma \alpha \beta \delta$ by (3.2) applied to $\alpha \beta \gamma$. Depending on $\beta$ and $\delta$ we get two sub-sub-sub-cases:

A. $\beta \geqslant \delta$. Then

$$
\begin{aligned}
\gamma \alpha \beta \delta & =c_{n} \gamma \alpha \delta \beta & & \text { (by (3.1) applied to } \alpha \beta \delta) \\
& =c_{n} \gamma \beta \alpha \delta . & & \text { (by (3.2) applied to } \alpha \delta \beta)
\end{aligned}
$$

Therefore $C_{D}\left(d_{\alpha \beta} d_{\gamma \delta}\right)=d_{\gamma \beta} d_{\alpha \delta}$. Since $\alpha>\gamma$, we have $d_{\alpha \beta} \succ_{\text {lex }} d_{\gamma \beta}$ and so $d_{\alpha \beta} d_{\gamma \delta} \succ_{\text {lex }} d_{\gamma \beta} d_{\alpha \delta}$.

B. $\delta>\beta$. Then $\gamma \alpha \beta \delta=c_{n} \gamma \delta \alpha \beta$ by (3.2) applied to $\alpha \beta \delta$. Therefore $\operatorname{CS}\left(d_{\alpha \beta} d_{\gamma \delta}\right)=d_{\gamma \delta} d_{\alpha \beta}$. Since $\alpha>\gamma$, we have $d_{\alpha \beta} \succ_{\text {lex }} d_{\gamma \delta}$ and so $d_{\alpha \beta} d_{\gamma \delta} \succ_{\text {lex }} d_{\gamma \delta} d_{\alpha \beta}$.

For any $w \in \mathrm{D}^{*}$, the word in $A^{*}$ that can be read from the subscripts represents the same element of $C_{n}$ as $w$. Since the original presentation (3.1)(3.2) is homogeneous, if $w, w^{\prime} \in \mathrm{D}^{*}$ are such that $w=\mathrm{C}_{n} w^{\prime}$, the words in $A^{*}$ read from the subscripts have the same length. So there are only finitely many possible words in $D^{*}$ representing each element of $C_{n}$. In particular, the $(A, \mathcal{T})$ is globally finite.

Notice that there is no word $w$ such that $w \succ_{\text {lex }} w$. Hence $(A, \mathcal{T})$ is acyclic. Since $(A, \mathcal{T})$ is globally finite and acyclic, it is noetherian.

Combining Lemmata 3.1 and 3.2 and the fact that D-Chinese staircase words form a language of unique normal forms for $C_{n}$, we obtain the desired result:

THEOREM 3.3. $(A, \mathcal{T})$ is a finite complete rewriting system presenting $C_{n}$.

\subsection{Algorithm for right-multiplication}

Cassaigne et al. $\left[\mathrm{CEK}^{+} \mathrm{OI}, \S 2.2\right]$ give the following algorithm that takes a symbol $\gamma \in A$ and the staircase $\sigma$ corresponding to a Chinese staircase word $w$ and computes the staircase corresponding to the unique staircase word equal to $w \gamma$. By starting with the empty staircase diagram (where all the entries are 0 , corresponding to the empty staircase word $\varepsilon$ ) and iteratively applying this algorithm, one can recover the staircase word equal in $C_{n}$ to an arbitrary word. For the purposes of describing this algorithm, the top $k$ rows of a staircase diagram form a k-staircase diagram. A k-staircase diagram corresponds to the k-staircase word $\ell^{(1)} \ldots \ell^{(k)}$, which is a prefix (not necessarily proper) of a staircase word (3.3). The algorithm will recursively work with a k-staircase diagram and a symbol $\gamma \leqslant k$; to start the computation, we simply begin with $k=n$. 


\section{Algorithm 3.4.}

Input: A k-staircase diagram $\sigma$ corresponding to a k-staircase word $\ell^{(1)} \ldots \ell^{(k)}$ and a symbol $\gamma \leqslant k$.

Output: A k-staircase diagram $\sigma \cdot \gamma$ corresponding to the k-staircase word equal to $\ell^{(1)} \ldots \ell^{(k)} \gamma$ in $C_{n}$.

Method: Write $\sigma=\left(\sigma^{\prime}, R_{1}\right)$, where $R_{1}$ is the bottom (k-th) row of the diagram and $\sigma^{\prime}$ the remaining $(k-1)$-staircase diagram.

1. If $\gamma=k$, then $\sigma \cdot \gamma=\left(\sigma^{\prime}, R_{1}^{\prime}\right)$, where $R_{1}^{\prime}$ is obtained from $R_{1}$ adding 1 to $\sigma_{\mathrm{k}}$.

2. If $\gamma<k$, let $\beta$ be maximal such that the entry in column $\beta$ of $R_{1}$ is non-zero. If no such $\beta$ exists, then set $\beta=\gamma$.

(a) If $\gamma \geqslant \beta$, then $\sigma \cdot \gamma=\left(\sigma^{\prime} \cdot \gamma, R_{1}\right)$.

(b) If $\gamma<\beta$ and $\beta<k$, then $\sigma \cdot \gamma=\left(\sigma^{\prime} \cdot \beta, R_{1}^{\prime}\right)$, where $R_{1}^{\prime}$ is obtained from $R_{1}$ by subtracting 1 from $\sigma_{k \beta}$ and adding 1 to $\sigma_{k \gamma}$.

(c) If $\gamma<\beta$ and $\beta=k$, then $\sigma \cdot \gamma=\left(\sigma^{\prime}, R_{1}^{\prime}\right)$ where $R_{1}^{\prime}$ is obtained from $R_{1}$ by subtracting 1 from $\sigma_{k}$ and adding 1 to $\sigma_{k \gamma}$.

\subsection{Algorithm for left-multiplication}

When we construct the biautomatic structure for the Chinese monoid and prove that the left-multiplication relation is recognized by a finite transducer, we will use the following left-handed analogue of Algorithm 3.4. We believe this algorithm is new and potentially of independent interest.

\section{Algorithm 3.5.}

Input: A Chinese staircase $\sigma$ corresponding to a Chinese staircase word $w$, and a symbol $\gamma \in A$.

Output: A Chinese staircase $\gamma \cdot \sigma$ corresponding to the unique Chinese staircase equal in $C_{n}$ to $\gamma w$.

Method: Store a symbol $\beta$ from $\{1, \ldots, n\} \cup\{\perp\}$, initially set to $\perp$. There are two stages. In the first stage, iterate the following for $\rho=1, \ldots, \gamma-1$ :

1. If every entry in row $\rho$ is empty, do nothing for this row.

2. Otherwise, let $\eta$ be the index of the rightmost non-zero entry (that is, $\eta$ is minimal with $\sigma_{\rho \eta}>0$ ). Then:

(a) If $\beta=\perp$ :

i. If $\eta<\rho$, decrement $\sigma_{\rho \eta}$ by 1 , increment $\sigma_{\rho}$ by 1 , and set $\beta=\eta$.

ii. If $\eta=\rho$, decrement $\sigma_{\rho}$ by 1 and set $\beta=\eta$.

(b) Otherwise, when $\beta \neq \perp$ :

i. If $\eta<\beta$, decrement $\sigma_{\rho \eta}$ by 1 , increment $\sigma_{\rho \beta}$ by 1 , and set $\beta=\eta$.

ii. Otherwise, when $\eta \geqslant \beta$, do nothing.

In the second stage, for $\rho=\gamma$,

1. If $\beta=\perp$, increment $\sigma_{\rho}$ by 1 .

2. Otherwise, when $\beta \neq \perp$, increment $\sigma_{\rho \beta}$ by 1 .

Finally, output the current staircase.

Proposition 3.6. Algorithm 3.5 always halts with the correct output. 
Proof of 3.6. First of all, notice that by setting $x=y$ in (3.2), we obtain

$$
x(z x)=c_{n}(z x) x ;
$$

and by setting $y=z$ in (3.1), we obtain

$$
z(z x)=\mathrm{c}_{\mathrm{n}}(z x) z,
$$

that is, $z x$ commutes with $x$ and with $z$ for $x \leqslant z$. Furthermore, for $w \leqslant x \leqslant$ $y \leqslant z$, by applying (3.2) twice we obtain

$$
(z w)(y x)=z w y x=c_{n} y z w x=c_{n} y x z w=(y x)(z w)
$$

that is, $z w$ and $y x$ commute for $w \leqslant x \leqslant y \leqslant z$.

We consider a staircase word (3.3) and a symbol $\gamma$ and the process of turning $\gamma \ell^{(1)} \ldots \ell^{(n)}$ into a staircase word $m^{(1)} \ldots m^{(n)}$ (where the $m^{(i)}$ are the row words) using the relations (3.1-3.2). Let $i$ be minimal such that $\ell^{(i)}$ is non-empty.

Depending on whether $i \geqslant \gamma$ or $i<\gamma$, we distinguish two cases. Suppose first that $i \geqslant \gamma$. Then for $\rho=1, \ldots, \gamma-1$, the iterative step in the algorithm always proceeds via case 1 , and these rows of the output staircase are the same as those of the input staircase. This is the correct output since

$$
\gamma \ell^{(1)} \cdots \ell^{(n)}=c_{n} \ell^{(1)} \cdots \ell^{(\gamma-1)} \gamma \ell^{(\gamma)} \cdots \ell^{(n)}
$$

When the algorithm reaches the step for $\rho=\gamma$, it still has $\beta=\perp$, and so it increments $\sigma_{\gamma}$ by 1 and halts. This produces a new word

$$
\mathrm{m}^{(\gamma)}=(\gamma 1)^{\sigma_{\gamma 1}} \cdots(\gamma(\gamma-1))^{\sigma_{\gamma(\gamma-1)}} \gamma^{\sigma_{\gamma}+1}
$$

that is equal in $C_{n}$ to $\gamma \ell^{(\gamma)}$, by repeated application of (3.6). Now, the resulting staircase word $m^{(1)} \cdots m^{(n)}$ is equal in $C_{n}$ to $\gamma \ell^{(1)} \cdots \ell^{(n)}$, since $m^{(\gamma)}=C_{n}$ $\gamma \ell^{(\gamma)}$ and $m^{(j)}=\ell^{(j)}$ for $j \neq \gamma$. Hence in this case the algorithm produces the correct output.

Suppose now that $i<\gamma$. Then the iterative step in the algorithm proceeds via case 1 for $\rho=1, \ldots, i-1$, and these rows of the output staircase are the same as those of the input staircase. That is, $m^{(j)}=\ell^{(j)}$ for $j=1, \ldots, i-1$. This is the correct output since

$$
\gamma \ell^{(1)} \cdots \ell^{(n)}=c_{n} \ell^{(1)} \cdots \ell^{(i-1)} \gamma \ell^{(i)} \cdots \ell^{(n)} .
$$

For $\rho=i$ the algorithm proceeds via case 2 (a). If $\eta<\rho$, then

$$
\begin{aligned}
& \gamma \ell^{(i)} \\
& =\gamma(i \eta)^{\sigma_{i \eta}} \ldots(i(i-1))^{\sigma_{i(i-1)}} i^{\sigma_{i}} \\
& =\gamma i \eta(i \eta)^{\sigma_{i \eta}-1} \cdots(i(i-1))^{\sigma_{i(i-1)} i^{\sigma_{i}}} \quad\left(\text { since } \sigma_{i \eta} \neq 0\right) \\
& =c_{n} \gamma \eta i(i \eta)^{\sigma_{i \eta}-1} \cdots(i(i-1))^{\sigma_{i(i-1)} i^{\sigma_{i}}} \quad(\text { by (3.1)) } \\
& =c_{n} \gamma \eta(i \eta)^{\sigma_{i \eta}-1} \cdots(i(i-1))^{\sigma_{i(i-1)} i^{\sigma_{i}+1}} \quad \text { (by (3.6)) } \\
& =c_{n}(i \eta)^{\sigma_{i \eta}-1} \cdots(i(i-1))^{\sigma_{i(i-1)} i^{\sigma_{i}+1}} \gamma \eta \quad\left(\text { by }(3.7) \text {, and (3.2) when } \sigma_{i}=0\right) \\
& =\mathrm{m}^{(\mathrm{i})} \gamma \eta
\end{aligned}
$$

and the algorithm, proceeding via case $2(a) i$, outputs the $i$-th row corresponding to $\mathrm{m}^{(\mathrm{i})}$ and now has $\beta=\eta$. (This parameter $\beta$ works as a letter that is 
'dragged' by $\gamma$ as it moves rightward through the word to its proper place.) If, on the other hand, $\eta=i$, then

$$
\begin{array}{rlrl} 
& \gamma \ell^{(i)} & \\
=\gamma i^{\sigma_{i}} & & \\
= & \gamma i i^{\sigma_{i}-1} & & \left(\text { since } \sigma_{i} \neq 0\right) \\
=c_{n} i^{\sigma_{i}-1} \gamma i, & & \left(\text { by }(3.7), \text { and }(3.2) \text { when } \sigma_{i}=2\right)
\end{array}
$$

and the algorithm, proceeding via case 2 (a)ii, outputs the $i$-th row corresponding to $m^{(i)}=i^{\sigma_{i}-1}$ and now has $\beta=i$. Notice that in either case we have $\gamma \ell^{(i)}=c_{n} m^{(i)} \gamma \beta$ and $\beta \leqslant \rho$, and so in the next iteration $\beta$ will be strictly less than the new value of $\rho$. We shall see by induction that $\beta$ is always less than $\rho$ when a new iteration begins.

For $\rho=i+1, \ldots, \gamma-1$, the algorithm produces empty rows whenever $\ell^{(\rho)}$ is empty and does not change $\beta$. So suppose $\ell^{(\rho)}$ is not empty. Then since $\beta \neq \perp$, the algorithm proceeds via case $2(b)$. We know that $\beta<\rho$. If $\eta<\beta$, then

$$
\begin{aligned}
& \gamma \beta \ell^{(\rho)} \\
& =\gamma \beta(\rho \eta)^{\sigma_{\rho \eta}} \cdots(\rho(\rho-1))^{\sigma_{\rho(\rho-1)}} \rho^{\sigma_{\rho}} \\
& =\gamma \beta \rho \eta(\rho \eta)^{\sigma_{\rho \eta}-1} \cdots(\rho(\rho-1))^{\sigma_{\rho(\rho-1)}} \rho^{\sigma_{\rho}} \\
& =\mathrm{C}_{n} \rho \gamma \beta \eta(\rho \eta)^{\sigma_{\rho \eta}-1} \cdots(\rho(\rho-1))^{\sigma_{\rho(\rho-1)}} \rho^{\sigma_{\rho}} \\
& =C_{n} \rho \gamma \eta \beta(\rho \eta)^{\sigma_{\rho \eta}-1} \cdots(\rho(\rho-1))^{\sigma_{\rho(\rho-1)}} \rho^{\sigma_{\rho}} \\
& =C_{n} \gamma \eta \rho \beta(\rho \eta)^{\sigma_{\rho \eta}-1} \cdots(\rho(\rho-1))^{\sigma_{\rho(\rho-1)}} \rho^{\sigma_{\rho}} \\
& =\mathrm{C}_{n} \rho \beta(\rho \eta)^{\sigma_{\rho \eta}-1} \cdots(\rho(\rho-1))^{\sigma_{\rho(\rho-1)}} \rho^{\sigma_{\rho}} \gamma \eta \\
& =c_{n}(\rho \eta)^{\sigma_{\rho \eta}-1} \cdots(\rho \beta)^{\sigma_{\rho \beta}+1} \cdots(\rho(\rho-1))^{\sigma_{\rho(\rho-1)}} \rho^{\sigma_{\rho}} \gamma \eta \quad \text { (by (3.7)), } \\
& =m^{(\rho)} \gamma \eta \text {, } \\
& \text { (since } \sigma_{\rho \eta-1} \neq 0 \text { ) } \\
& \text { (by (3.2)) } \\
& \text { (by (3.7), and (3.2) for } \sigma_{\rho}=1 \text { ) }
\end{aligned}
$$

and the algorithm, proceeding via case $2(b) \mathrm{i}$, outputs the $\rho$-th row corresponding to $m^{(\rho)}$ and now has $\beta=\eta$. If, on the other hand, $\eta \geqslant \beta$, then

$$
\begin{aligned}
& \gamma \beta \ell^{(\rho)} \\
&= \gamma \beta(\rho \eta)^{\sigma_{\rho \eta}} \cdots(\rho(\rho-1))^{\sigma_{\rho(\rho-1)}} \rho^{\sigma_{\rho}} \\
&=\mathrm{c}_{n}(\rho \eta)^{\sigma_{\rho \eta}} \cdots(\rho(\rho-1))^{\sigma_{\rho(\rho-1)}} \rho^{\sigma_{\rho}} \gamma \beta \quad\left(\text { by }(3.7), \text { and }(3.2) \text { when } \sigma_{\rho}=1\right) \\
&=m^{(\rho)} \gamma \beta .
\end{aligned}
$$

and the algorithm, proceeding via case $2(b)$ ii, outputs the same $\rho$-th row (corresponding to $m^{(\rho)}=\ell^{(\rho)}$ ) and has $\beta$ unchanged. Notice that in either case $\gamma \beta \ell^{(\rho)}=C_{n} m^{(\rho)} \gamma \hat{\beta}$, where $\hat{\beta}$ is the new value of $\beta$ obtained by executing the algorithm. Also, $\beta \leqslant \rho$, and so in the next iteration $\beta$ will be strictly less than the new value of $\rho$.

Finally, for $\rho=\gamma$,

$$
\begin{array}{rlrl} 
& \gamma \beta \ell^{(\rho)} & \\
= & \rho \beta(\rho \eta)^{\sigma_{\rho \eta}} \cdots(\rho(\rho-1))^{\sigma_{\rho(\rho-1)}} \rho^{\sigma_{\rho}} & & (\text { since } \gamma=\rho) \\
= & c_{n}(\rho \eta)^{\sigma_{\rho \eta}} \cdots(\rho \beta)^{\sigma_{\rho \beta}+1} \cdots(\rho(\rho-1))^{\sigma_{\rho(\rho-1)}} \rho^{\sigma_{\rho}} & & (\text { by }(3.7)), \\
= & m^{(\rho)} &
\end{array}
$$


and so the algorithm, proceeding via case 2 , outputs the $\rho$-th row corresponding to $\mathrm{m}^{(\rho)}$ and halts.

For $j>\rho$, we have $\ell^{(j)}=m^{(j)}$ and so, at the end of the algorithm, we have the staircase diagram corresponding to the Chinese staircase word $m^{(1)} \cdots m^{(n)}$.

\subsection{Biautomaticity}

The essential idea in constructing our biautomatic structure is to have a language of normal forms related to (3.3) and to show that Algorithms 3.4 and 3.5 can be performed by a transducer. We will initially use the generating set $D$ introduced in $\S 3.2$, but we will later switch to the standard generating set $A$.

Let

$$
K^{(k)}=d_{k 1}^{*} d_{k 2}^{*} \cdots d_{k(k-1)}^{*} d_{k}^{*}
$$

and

$$
K=K^{(1)} K^{(2)} \cdots K^{(n-1)} K^{(n)} ;
$$

notice that $\mathrm{K}$ is regular.

\subsubsection{Right-multiplication by transducer}

Let us show that the relation $\mathrm{K}_{\mathrm{d}_{\gamma}}=\left\{(\mathrm{u}, v): \mathrm{u}, \boldsymbol{v} \in \mathrm{K}, \mathrm{ud} \mathrm{d}_{\gamma}=\mathrm{C}_{\mathrm{n}} v\right\}$ is recognized by a finite transducer for any $\gamma \in\{1, \ldots, n\}$. We only need to consider right multiplication by $d_{\gamma}$, where $\gamma \in A$, because we will later switch back to the generating set $A$.

We imagine a transducer reading a pair of words in $\mathrm{K}$ from right to left with the aim of checking whether this pair is in $\mathrm{K}_{\mathrm{d}_{\gamma}}$. It is easiest to describe the transducer as reading symbols from the top tape and outputting symbols on the bottom tape. As it reads a symbol from the top tape, the transducer knows which language $K^{(k)}$ (corresponding to the k-th row) the current symbol comes from, for symbols $d_{k \beta}$ and $d_{k}$ occur only in subwords from $k^{(k)}$. The transducer stores a symbol $\alpha$ from $\{1, \ldots, n\} \cup\{\infty\}$ that records the symbol to be inserted into the $(k-1)$-staircase word from $K^{(1)} \cdots K^{(k-1)}$. Initially $\alpha=\gamma$. The value $\alpha=\infty$ indicates that no further insertion is necessary. In this case the transducer simply reads symbols from the top tape and outputs the same symbols on the bottom tape until the end of input.

When $\alpha \neq \infty$, the transducer functions as follows. As remarked above, the transducer knows when it starts reading a subword from $K^{(k)}$. If $\alpha=k$, the transducer outputs an extra symbol $d_{k}$ (corresponding to case 1 in Algorithm 3.4) and sets $\alpha=\infty$. If $\alpha<k$, the transducer needs to calculate $\beta$ in accordance with case 2 of Algorithm 3.4. But $\beta$ can be determined from the rightmost symbol of the subword from $K^{(k)}$, which is the first symbol of this subword it encounters, since it is reading right-to-left: this symbol is $d_{k \beta}$ or $d_{k}$, in which case $\beta=k$. [If there is no symbol from $K^{(k)}$ on the top tape, then the next symbol it reads is from $K^{(j)}$ for some $j<k$. No special action is required here, for the automaton now proceeds to insert $\alpha$ into the subword from $K^{(1)} \ldots K^{(j)}$, which is in accordance with the recursion in case 2(a) of Algorithm 3.4.]

If $\alpha \geqslant \beta$, the transducer reads each symbol of the subword from $K^{(k)}$ and outputs it on the bottom tape, all the while keeping the same value of $\alpha$. It 
then arrives at the rightmost end of the next subword with the correct value of $\alpha$ (this corresponds to the recursion in case 2(a) of Algorithm 3.4).

If $\alpha<\beta$ and $\beta<k$, then the transducer reads each symbol of the subword from $\mathrm{K}^{(\mathrm{k})}$ and outputs it on the bottom tape, with the following exceptions:

- It does not output anything on reading the first symbol $d_{k \beta}$ (corresponding to decrementing $\sigma_{k \beta}$ by 1 ).

- It outputs an extra symbol $d_{k \alpha}$ immediately after reaching the leftmost end of the (possibly empty) subword of symbols $d_{k \alpha}$ (it can non-deterministically predict when it has reached the end of this subword; this corresponds to incrementing $\sigma_{\mathrm{k} \alpha}$ by 1 ).

On reaching the end of the subword from $\mathrm{K}^{(\mathrm{k})}$, it sets $\alpha=\beta$, corresponding to the recursion in case 2(b) of Algorithm 3.4).

If $\alpha<\beta$ and $\beta=k$, then the transducer reads each symbol of the subword from $\mathrm{K}^{(\mathrm{K})}$ and outputs it on the bottom tape, with the following exceptions:

- It does not output anything on reading the first symbol $d_{k}$ (corresponding to decrementing $\sigma_{k}$ by 1 ).

- It outputs an extra symbol $d_{k \alpha}$ immediately after reaching the leftmost end of the (possibly empty) subword of symbols $d_{k \alpha}$ (it can non-deterministically predict when it has reached the end of this subword; this corresponds to incrementing $\sigma_{\mathrm{k} \alpha}$ by 1 ).

On reaching the end of the subword from $\mathrm{K}^{(\mathrm{k})}$, it sets $\alpha=\infty$, corresponding to case 2(c) of Algorithm 3.4).

This shows that $K_{d_{\gamma}}$ is recognized by a transducer; $K_{d_{\gamma}}$ is therefore a rational relation.

\subsubsection{Left-multiplication by transducer}

Let us show that the relation $\mathrm{d}_{\gamma} \mathrm{K}=\left\{(\mathrm{u}, v): \mathrm{u}, v \in \mathrm{K}, \mathrm{d}_{\gamma} \mathrm{u}=\mathrm{C}_{\mathrm{n}} v\right\}$ is recognized by a finite transducer for any $\gamma \in\{1, \ldots, n\}$.

We imagine a transducer reading a pair of words in $\mathrm{K}$ from left to right and checking whether this pair is in $\mathrm{d}_{\gamma} \mathrm{K}$. As before, we will describe the transducer as reading symbols from the top tape and outputting symbols on the bottom tape. As it reads a symbol from the top tape, the transducer knows which $K^{(k)}$ the current symbol comes from, for symbols $d_{k \alpha}$ and $d_{k}$ occur only in subwords from $K^{(k)}$. The transducer also non-deterministically looks ahead one symbol on the top tape, so it knows when it has reached the end of a substring (possibly empty) of symbols $d_{k \alpha}$ or $d_{k}$.

In its state, the transducer stores the symbol $\beta \in\{1, \ldots, n\} \cup\{\perp, \infty\}$. Initially $\beta=\perp$. The value $\beta=\infty$ indicates that no further changes are necessary and the transducer simply reads symbols from the top tape and outputs the same symbols on the bottom tape. When $\beta \neq \infty$, the transducer functions as follows.

Suppose it is reading some subword from $K^{(\rho)}$ from the top tape, where $\rho<\gamma$. If this subword is empty, then the corresponding output subword is empty, as per case 1 of the iterative part of Algorithm 3.5. When this subword is non-empty, and the algorithm proceeds via case $2(a)$ or $2(b)$, the transducer can determine $\eta$ from the first symbol $d_{\rho \eta}$ or $d_{\rho}$ of the subword. It reads each symbol of this subword and outputs the same symbol with the following exceptions: 
- If $\beta=\perp$ and $\eta<\rho$ (case 2(a)i), it outputs $\varepsilon$ on reading this symbol $d_{\rho \eta}$ (corresponding to decrementing $\sigma_{\rho \eta}$ by 1 ), outputs an extra symbol $d_{\rho}$ immediately after reading the last symbol of this subword (corresponding to incrementing $\sigma_{\rho}$ by 1$)$, and sets $\beta=\eta$.

- If $\beta=\perp$ and $\eta=\rho$ (case 2(a)ii), it outputs $\varepsilon$ on reading the first symbol $d_{\rho}$ (corresponding to decrementing $\sigma_{\rho}$ by 1 ), and sets $\beta=\eta$.

- If $\beta \neq \perp$ and $\eta<\beta$ (case 2(b)i), it outputs $\varepsilon$ on reading this symbol $d_{\rho \eta}$ (corresponding to decrementing $\sigma_{\rho \eta}$ by 1 ), outputs an extra symbol $d_{\rho \beta}$ immediately after reading the last symbol $d_{\rho \beta}$ (corresponding to incrementing $\sigma_{\rho \beta}$ by 1 ), and sets $\beta=\eta$.

[Notice that in case $2(\mathrm{~b}) \mathrm{ii}$, the transducer simply reads symbols and outputs them: there are no exceptions in this case.]

Finally, on reading the subword from $K^{(\gamma)}$, again it reads symbols and outputs them, except that if $\beta=\perp$ it outputs an extra symbol $d_{\gamma}$ at the end of the (possibly empty) string of symbols $d_{\gamma}$, and if $\beta \neq \perp$, it outputs an extra symbol $d_{\gamma \beta}$ at the end of the (possibly empty) string of symbols $d_{\gamma \beta}$. On doing this, it sets $\beta=\infty$ and simply reads to the end of the top tape and outputs the same symbols on the bottom tape.

This shows that $\mathrm{d}_{\gamma} \mathrm{K}$ is recognized by a transducer; $\mathrm{d}_{\gamma} \mathrm{K}$ is therefore a rational relation.

\subsubsection{Deducing biautomaticity}

Let $\mathcal{Q} \subseteq \mathrm{D}^{*} \times A^{*}$ be the rational relation

$$
\left\{\left(d_{\alpha \beta}, \alpha \beta\right),\left(d_{\alpha}, \alpha\right): \alpha, \beta \in A, \alpha>\beta\right\}^{*} .
$$

Let

$$
\mathrm{L}=\mathrm{K} \circ \mathrm{Q}=\left\{\mathrm{u} \in \mathrm{A}^{*}:\left(\exists \mathrm{u}^{\prime} \in \mathrm{K}\right)\left(\left(\mathrm{u}^{\prime}, \mathrm{u}\right) \in \mathcal{Q}\right)\right\} ;
$$

then $L$ is a regular language over $A$ that maps onto $C_{n}$. [In fact, $L$ is the set of Chinese staircase words, but we do not need to know this.] Then for any $\gamma \in A$,

$$
\begin{aligned}
(\mathrm{u}, v) \in \mathrm{L}_{\gamma} & \Longleftrightarrow \mathrm{u} \in \mathrm{L} \wedge v \in \mathrm{L} \wedge \mathrm{u} \gamma=\mathrm{c}_{\mathrm{n}} v \\
& \Longleftrightarrow\left(\exists \mathrm{u}^{\prime}, v^{\prime} \in \mathrm{K}\right)\left(\left(\mathrm{u}^{\prime}, \mathrm{u}\right) \in \mathcal{Q} \wedge\left(v^{\prime}, v\right) \in \mathcal{Q} \wedge \mathrm{u}^{\prime} \mathrm{d}_{\gamma}=\mathrm{C}_{\mathrm{n}} v^{\prime}\right) \\
& \Longleftrightarrow\left(\exists \mathrm{u}^{\prime}, v^{\prime} \in \mathrm{K}\right)\left(\left(\mathrm{u}^{\prime}, \mathrm{u}\right) \in \mathcal{Q} \wedge\left(v^{\prime}, v\right) \in \mathcal{Q} \wedge\left(\mathrm{u}^{\prime}, v^{\prime}\right) \in \mathrm{K}_{\mathrm{d}_{\gamma}}\right) \\
& \Longleftrightarrow(\mathrm{u}, v) \in \mathcal{Q}^{-1} \circ \mathrm{K}_{\mathrm{d}_{\gamma}} \circ \mathcal{Q} .
\end{aligned}
$$

Therefore $L_{\gamma}$ is a rational relation. Now, if $(u, v) \in L_{\gamma}$, then $|v|=|u|+1$ since the defining relations (3.1-3.2) preserve lengths of words over $A$. By Proposition 2.3, $\mathrm{L}_{\gamma} \delta_{\mathrm{R}}$ and $\mathrm{L}_{\gamma} \delta_{\mathrm{L}}$ are regular.

Similarly, from the fact that ${ }_{c_{\gamma}} \mathrm{K}$ is a rational relation, we deduce that ${ }_{\gamma} \mathrm{L}=$ $Q^{-1} \circ \mathrm{d}_{\gamma} \mathrm{K} \circ \mathcal{Q}$ is a rational relation and thus, by Proposition 2.3, that $\gamma \mathrm{L} \delta_{\mathrm{R}}$ and ${ }_{\gamma} \mathrm{L} \delta_{\mathrm{L}}$ are regular.

This proves the result:

TheOREm 3.7. $(A, L)$ is a biautomatic structure for the Chinese monoid $C_{n}$. 


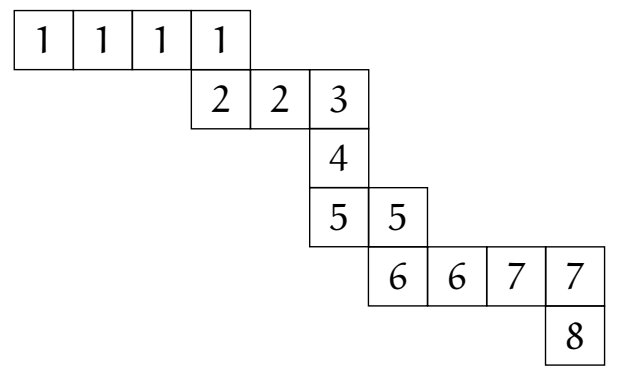

FIGURE 1. Example of a quasi-ribbon tableau.

\section{HYPOPLACTIC MONOID}

\subsection{Quasi-ribbon tableaux}

Let $n \in \mathbb{N}$. Let $A$ be the ordered alphabet $\{1<2<\ldots<n\}$. Let $\mathcal{R}$ be the set of defining relations for the Plactic monoid; that is,

$$
\mathcal{R}=\{(\mathrm{acb}, \mathrm{cab}): \mathrm{a} \leqslant \mathrm{b}<\mathrm{c}\} \cup\{(\mathrm{bac}, \mathrm{bca}): \mathrm{a}<\mathrm{b} \leqslant \mathrm{c}\} .
$$

and let

$$
\mathcal{S}=\{(\mathrm{cadb}, \mathrm{acbd}),(\mathrm{bdac}, \mathrm{dbca}): \mathrm{a} \leqslant \mathrm{b}<\mathrm{c} \leqslant \mathrm{d}\} .
$$

The hypoplactic monoid of rank $n$, denoted $H_{n}$, is presented by $\langle A \mid \mathcal{R} \cup \mathcal{S}\rangle$.

A column is a strictly decreasing word in $A^{*}$ (that is, a word $\alpha=\alpha_{1} \cdots \alpha_{k}$, where $\alpha_{i} \in A$, such that $\alpha_{i}>\alpha_{i+1}$ for all $\left.i=1, \ldots, k-1\right)$. Any word $\alpha \in A^{*}$ has a decomposition as a product of columns of maximal length $\alpha=$ $\alpha^{(1)} \ldots \alpha^{(k)}$. Such a word $\alpha$ is a quasi-ribbon word if the last (smallest) symbol of $\alpha^{(i+1)}$ is greater than or equal to the first (greatest) symbol of $\alpha^{(i)}$ for all $i=1, \ldots, k-1$.

For example, 111212543656787 is a quasi-ribbon word. (For clarity, spaces indicate the decomposition into columns of maximum length.) Any quasi-ribbon word has a planar representation as a quasi-ribbon tableau, where the columns are written vertically from bottom to top and arranged left to right so that the last (uppermost) symbol in each column aligns with the first symbol of the previous column. The quasi-ribbon tableau corresponding to 111212543656787 is shown in Figure 1; notice that each row in the quasi-ribbon tableau is non-decreasing.

Theorem 4.1 ([Novoo, Theorem 4.17]). The quasi-ribbon words form a crosssection of the hypoplactic monoid.

For any $w \in A^{*}$, let $Q(w)$ denote the unique quasi-ribbon word such that $w=\mathrm{H}_{\mathrm{n}} \mathrm{Q}(w)$.

TheOREM 4.2 ([Novoo, Theorem 5.12]). For any $w \in A^{*}$, the quasi-ribbon word $\mathrm{Q}(w)$ is the lexicographically minimum word such that $w=\mathrm{H}_{\mathrm{n}} \mathrm{Q}(w)$. That is, $\mathrm{Q}(w) \leqslant_{\text {lex }} w$ for all $w \in A^{*}$.

Algorithm 4.3 ([Novoo, Algorithm 4.4]).

Input: A quasi-ribbon word $w$ and a symbol a.

Output: The quasi-ribbon word $\mathrm{Q}(w \mathrm{w})$.

Method: 
Let $\mathrm{T}$ be the quasi-ribbon tableau corresponding to $w$. If there is no entry in $T$ that is less than or equal to $a$, output the word corresponding to the tableau obtained by putting $a$ and gluing $T$ to the bottom of $a$.

Otherwise, let $x$ be the right-most and bottom-most entry of $T$ that is less than or equal to $x$. Put a new entry a to the right of $x$ and glue the remaining part of $T$ (below and to the right of $x$ ) onto the bottom of the new entry $a$. Output the quasi-ribbon word corresponding to the new tableau.

The symmetry of the presentation $\langle A \mid \mathcal{R} \cup \mathcal{S}\rangle$ and of the definition of quasiribbon words means that Algorithm 4.3 has the following symmetrical version, describing how to left-multiply a quasi-ribbon word by a generator.

\section{Algorithm 4.4.}

Input: A quasi-ribbon word $w$ and a symbol a.

Output: The quasi-ribbon word $\mathrm{Q}(\mathrm{a} w)$.

Method:

Let $\mathrm{T}$ be the quasi-ribbon tableau corresponding to $w$. If there is no entry in $T$ that is greater than or equal to $a$, output the word corresponding to the tableau obtained by putting $a$ and gluing $T$ to the top of $a$.

Otherwise, let $x$ be the left-most and upper-most entry of $T$ that is greater than or equal to $x$. Put a new entry a to the left of $x$ and glue the remaining part of $T$ (above and to the left of $x$ ) onto the top of the new entry a. Output the quasi-ribbon word corresponding to the new tableau.

\subsection{Finite complete rewriting system}

Let $\mathcal{T}=\left\{w \rightarrow Q(w): w \in A^{*} \wedge w \neq Q(w) \wedge|w| \leqslant \max \{2 n, 4\}\right\}$.

THEOREM 4.5. (A, T) is a finite complete rewriting system presenting $\mathrm{H}_{n}$.

Proof of 4.5. First of all notice that every rules in $\mathcal{T}$ holds in $\mathrm{H}_{\mathrm{n}}$ and thus is a consequence of the relations in $\mathcal{R} \cup \mathcal{S}$. On the other hand, every relation in $\mathcal{R} \cup \mathcal{S}$ is a consequence of the rules in $\mathcal{T}$ (since $\mathcal{T}$ includes all rules $w \rightarrow Q(w)$ for all $|w| \leqslant 4)$. Hence $\langle A \mid \mathcal{T}\rangle$ presents $H_{n}$.

Next, notice that there are only finitely many rules in $\mathcal{T}$ since there are only finitely many possibilities for $w$ and $\mathrm{Q}(w)$ is uniquely determined.

Suppose $u \rightarrow \mathcal{T} v$. Then $|u|=|v|$ and $v<_{\operatorname{lex}} u$ by Theorem 4.2 (since a rule $w \rightarrow \mathrm{Q}(w)$ is applied where $w \neq \mathrm{Q}(w)$ and $\mathrm{Q}(w) \leqslant \operatorname{lex} w$ for all $\left.w \in A^{*}\right)$. Hence $(A, \mathcal{T})$ is acyclic. Furthermore, since $|w|=|Q(w)|$, rewriting using $(A, \mathcal{T})$ preserves lengths, and so for each $u \in A^{*}$, there are only finitely many words $v$ such that $u \rightarrow_{\mathcal{T}}^{*} v$; thus $(A, \mathcal{T})$ is globally finite. Hence $(A, \mathcal{T})$ is noetherian.

Let $u \in A^{*}$. Since $(A, \mathcal{T})$ is noetherian, applying rewriting rules from $\mathcal{T}$ to $u$ will always yield an irreducible word $v$.

With the aim of obtaining a contradiction, suppose that $v$ is not a quasiribbon word. Let $v=v^{(1)} v^{(2)} \cdots v^{(\mathrm{k})}$ be the decomposition of $v$ into columns of maximum length. Since $v$ is not a quasi-ribbon word, for some $i$ the word $v^{(i)} v^{(i+1)}$ is not a quasi-ribbon word. Since columns are strictly descending words, they have maximum length $n$. So $v^{(i)} v^{(i+1)}$ has length at most $2 n$. Since $v^{(i)} v^{(i+1)}$ is not a quasi-ribbon word, $v^{(i)} v^{(i+1)} \neq \mathrm{Q}\left(v^{(i)} v^{(i+1)}\right)$. Hence $v^{(i)} v^{(i+1)} \rightarrow \mathrm{Q}\left(v^{(i)} v^{(i+1)}\right)$ is a rule in $\mathcal{T}$. Since $v$ contains the left-hand side of this rule, it is not irreducible, which is a contradiction. This proves that $v$ is a quasi-ribbon word. 
Hence the set of irreducible words for $(A, \mathcal{T})$ are the quasi-ribbon words, which form a cross-section of $H_{n}$ by Theorem 4.1. Hence $(A, \mathcal{T})$ is confluent.

\section{$4 \cdot 3$ Biautomaticity}

\subsubsection{Right-and left-multiplication by transducer}

Let

$$
\mathrm{C}=\left\{\mathrm{c}_{\alpha}: \alpha \in \mathrm{A}^{+} \text {is a column }\right\} .
$$

Each symbol $c_{\alpha}$ represents the element $\alpha$ of $\mathrm{H}_{\mathrm{n}}$. Since this holds in particular when $|\alpha|=1$, the original generating set $A$ is essentially contained in $C$. Thus $C$ also generates $H_{n}$.

Define a relation $\preceq$ on columns by $\alpha \preceq \beta$ if the first (greatest) symbol of $\alpha$ is less than or equal to the last (smallest) symbol of $\beta$. That is, $\alpha \preceq \beta$ if $\alpha$ can appear immediately to the left of $\beta$ in the decomposition of a quasi-ribbon word into maximal columns.

Now let

$$
K=\left\{c_{\alpha^{(1)}} c_{\alpha^{(2)}} \cdots c_{\alpha^{(k)}}: k \in \mathbb{N} \cup\{0\}, c_{\alpha^{(i)}} \in C, \alpha^{(j)} \preceq \alpha^{(j+1)} \text { for all } j\right\} .
$$

Notice that $\mathrm{K}$ is regular since an automaton checking whether $\mathrm{c}_{\alpha^{(1)}} \mathrm{c}_{\alpha^{(2)}} \cdots \mathrm{c}_{\alpha^{(\mathrm{k})}} \in$ $\mathrm{C}^{*}$ lies in $\mathrm{K}$ need only store the previous letter in its state in order to check whether $\alpha^{(j)} \preceq \alpha^{(j+1)}$ for all $j$.

We will first of all prove that for any $\gamma \in A$ the relation $K_{c_{\gamma}}$ is recognized by a finite transducer.

We imagine a transducer reading a pair of words

$$
\left(c_{\alpha^{(1)}} \cdots c_{\alpha^{(k)}}, c_{\beta^{(1)}} \cdots c_{\beta^{(1)}}\right) \in K \times K
$$

from right to left, with the aim of checking whether this pair is in $\mathrm{K}_{\mathrm{c}_{\gamma}}$. It is easiest to describe the transducer as reading symbols from the top tape and outputting symbols on the bottom tape. Essentially, the transducer will perform Algorithm 4.3 using the alphabet $C$ to store columns quasi-ribbon words.

The transducer non-deterministically looks one symbol ahead (that is, further left) on the top tape. In its state it stores either $\gamma$ or $\infty$, with the latter indicating that $\gamma$ has already been inserted into the quasi-ribbon.

While the transducer is storing $\gamma$ in its state, it examines each column $c_{\alpha^{(j)}}$ it reads and proceeds as follows:

- If $\gamma$ is less than every symbol of $\alpha^{(j)}$ and $j>1$ and $\gamma$ is less than the first symbol of $\alpha^{(j-1)}$, then the transducer outputs $c_{\alpha^{(j)}}$ and proceeds to read the next symbol $c_{\alpha^{(j-1)}}$. (Notice that the transducer knows whether $j>1$ since it non-deterministically looks ahead one symbol.)

- If $\gamma$ is greater than or equal to some symbol of $\alpha^{(j)}$, then the transducer outputs $c_{\beta^{\prime}} c_{\beta \gamma}$, where $\alpha^{(j)}=\beta \beta^{\prime}$ and the first letter of $\beta^{\prime}$ is the first symbol of $\alpha^{(j)}$ that is less than or equal to $\gamma$. It then stores $\infty$ in its state in place of $\gamma$.

- If $\gamma$ is less than every symbol of $\alpha^{(j)}$ and either $j=1$ or $j>1$ and $\gamma$ is greater than or equal to the first symbol of $\alpha^{(j-1)}$, then the transducer outputs $c_{\alpha^{(j)} \gamma}$. It then stores $\infty$ in its state in place of $\gamma$. 
When the transducer is storing $\infty$ in its state, it simply reads every input symbol and outputs the same symbol until it reaches the end of the input.

Finally, if the top tape is the empty word $\varepsilon$, the transducer simply outputs $\gamma$.

Since $\mathrm{K}_{\mathrm{c}_{\gamma}}$ is recognized by a finite transducer, it is a rational relation.

Since Algorithms 4.3 and 4.4 are symmetric, it is clear that ${ }_{c_{\gamma}} \mathrm{K}$ is also recognized by a finite transducer (which effectively performs Algorithm 4.4 using the alphabet $\mathrm{C}$ to store column quasi-ribbon words). So ${ }_{c_{\gamma}} \mathrm{K}$ is a rational relation.

\subsubsection{Deducing biautomaticity}

Let $Q \subseteq C^{*} \times A^{*}$ be the relation

$$
\left\{\left(c_{\alpha^{(1)}} c_{\alpha^{(2)}} \cdots c_{\alpha^{(k)}}, \alpha^{(1)} \alpha^{(2)} \cdots \alpha^{(k)}\right): k \in \mathbb{N} \cup\{0\} \text {, each } \alpha^{(i)} \text { is a column }\right\} .
$$

It is easy to see that $Q$ is a rational relation. Let

$$
\mathrm{L}=\mathrm{K} \circ \mathcal{Q}=\left\{\mathrm{u} \in \mathrm{A}^{*}:\left(\exists \mathrm{u}^{\prime} \in \mathrm{K}\right)\left(\left(\mathrm{u}^{\prime}, \mathrm{u}\right) \in \mathcal{Q}\right)\right\} .
$$

Then $L$ is a regular language over $A$ that maps onto $M_{n}$, since the set of regular languages is closed under applying rational relations. (In fact, L is the set of quasi-ribbon words, but this is not important for proving biautomaticity.) Then for any $\gamma \in A$,

$$
\begin{aligned}
(\mathrm{u}, v) \in \mathrm{L}_{\gamma} & \Longleftrightarrow \mathrm{u} \in \mathrm{L} \wedge v \in \mathrm{L} \wedge \mathrm{u} \gamma=\mathrm{H}_{\mathrm{n}} v \\
& \Longleftrightarrow\left(\exists \mathrm{u}^{\prime}, v^{\prime} \in \mathrm{K}\right)\left(\left(\mathrm{u}^{\prime}, \mathrm{u}\right) \in \mathcal{Q} \wedge\left(v, v^{\prime}\right) \in \mathcal{Q} \wedge \mathrm{u}^{\prime} \mathrm{c}_{\gamma}=_{\mathrm{H}_{\mathrm{n}}} v^{\prime}\right) \\
& \Longleftrightarrow\left(\exists \mathrm{u}^{\prime}, v^{\prime} \in \mathrm{K}\right)\left(\left(\mathrm{u}^{\prime}, \mathrm{u}\right) \in \mathcal{Q} \wedge\left(v, v^{\prime}\right) \in \mathcal{Q} \wedge\left(\mathrm{u}^{\prime}, v^{\prime}\right) \in \mathrm{K}_{\mathrm{c}_{\gamma}}\right) \\
& \Longleftrightarrow(\mathrm{u}, v) \in \mathcal{Q}^{-1} \circ \mathrm{K}_{\mathrm{c}_{\gamma}} \circ \mathcal{Q} .
\end{aligned}
$$

Therefore, $\mathrm{L}_{\gamma}$ is a rational relation. Now, if $(u, v) \in \mathrm{L}_{\gamma}$, then $|v|=|u|+1$ since $u \gamma={ }_{H_{n}} v$ and the defining relations $\mathcal{R} \cup \mathcal{S}$ preserve lengths of words. By Proposition 2.3, $\mathrm{L}_{\gamma} \delta_{\mathrm{R}}$ and $\mathrm{L}_{\gamma} \delta_{\mathrm{L}}$ are regular.

Similarly, from the fact that ${ }_{c_{\gamma}} \mathrm{K}$ is a rational relation, we deduce that ${ }_{\gamma} \mathrm{L}=$ $Q^{-1} \circ \mathrm{d}_{\gamma} \mathrm{K} \circ \mathrm{Q}$ is a rational relation and thus, by Proposition 2.3, that ${ }_{\gamma} \mathrm{L} \delta_{\mathrm{R}}$ and ${ }_{\gamma} \mathrm{L} \delta_{\mathrm{L}}$ are regular.

Thus we have proved the desired result:

THeOREM 4.6. (A, L) is a biautomatic structure for the hypoplactic monoid $\mathrm{H}_{n}$.

\section{SYLVESTER MONOID}

Let $n \in \mathbb{N}$. Let $A$ be the finite ordered alphabet $\{1<2<\ldots<n\}$. Let $\mathcal{R}$ be the (infinite) set of defining relations

$$
\left\{(c a v b, a c v b): a \leqslant b<c, v \in A^{*}\right\} .
$$

Then the sylvester monoid of rank $n$, denoted $S_{n}$, is presented by $\langle A \mid \mathcal{R}\rangle$. This is simply a restriction to finite rank of the sylvester monoid as defined by Hivert et al. [HNTo5, Definition 8]. 


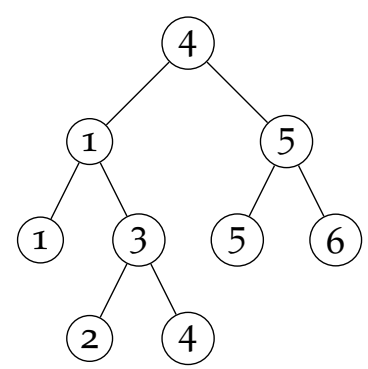

FIGURE 2. Example of a binary search tree T. The root has label 4, so every label in the left subtree of the root is less than or equal to 4 (and indeed the label 4 does occur) and every label in the right subtree of the root is strictly greater than 4. Notice that (for example) $T=\mathcal{T}(265415314$ ), and that $\operatorname{LRP}(T)=124315654$.

\subsection{Complete rewriting system}

The aim of this section is to prove that $(A, \mathcal{R})$ is a complete rewriting system. The proof will depend on two results proved by Hivert et al. (Propositions 5.1 and 5.2 below), and we need to define some new concepts first.

A (right strict) binary search tree is a labelled rooted binary tree where the label of each node is greater than or equal to the label of every node in its left subtree, and strictly less than every node in its right subtree; see the example in Figure 2.

Given a binary search tree $T$ and a symbol $a \in A$, one inserts a into $T$ as follows: if $T$ is empty, create a node and label it a. If $T$ is non-empty, examine the label $x$ of the root node; if $a \leqslant x$, recursively insert $a$ into the left subtree of the root node; otherwise recursively insert a into the right subtree of the root note. Denote the resulting tree $a \cdot T$. It is easy to see that $a \cdot T$ is also a binary search tree.

Given any word $w \in A^{*}$, define its corresponding binary search tree $\mathcal{T}(w)$ as follows: start with the empty tree and iteratively insert the symbols in $w$ from right to left; again, see the example in Figure 2.

The left-to-right postfix reading $\operatorname{LRP}(\mathrm{T})$ of a binary search tree $\mathrm{T}$ is defined to be the word obtained as follows: recursively perform the left-to-right postfix reading of the left subtree of the root of $\mathrm{T}$, then recursively perform the left-toright postfix reading of the right subtree of the root of $T$, then output the label of the root of $T$; again, see the example in Figure 2. Note that $\mathcal{T}(\operatorname{LRP}(T))=T$.

Proposition 5.1 ([HNTo5, Theorem 10]). Let $w, w^{\prime} \in A^{*}$. Then $w=s_{n} w^{\prime}$ if and only if $\mathcal{T}(w)=\mathcal{T}\left(w^{\prime}\right)$.

Proposition 5.2 ([HNTo5, Proposition 15]). Let $w \in A^{*}$. Then $\operatorname{LRP}(\mathcal{T}(w))$ is the lexicographically minimal word representing the same element of $S_{n}$ as $w$.

Proposition 5.3. ( $A, \mathcal{R})$ is a complete rewriting system, and the irreducible words are the lexicographically minimal words representing elements of $S_{n}$.

Proof of 5.3. Notice that an application of a rewriting rule from $\mathcal{R}$ strictly decreases a word with respect to the lexicographic order. Hence $(A, \mathcal{R})$ is acyclic. Since it does not alter the length of a word, and since there are only finitely many words of a given length, $(A, \mathcal{R})$ is globally finite. Hence $(A, \mathcal{R})$ is noetherian. 
To see that $(A, \mathcal{R})$ is confluent, we prove that the irreducible words are precisely those words that arise as left-to-right postfix readings of binary search trees.

First, let us show that any word that arises as a left-to-right-postfix reading of a binary search tree must be irreducible. Let $w$ be a left-to-right postfix reading of some binary search tree. That is, $w=\operatorname{LRP}(\mathcal{T}(w))$. Then by Proposition $5.2, w$ is the lexicographically minimal word among all words representing the same element of $S_{n}$ as $w$. Since an application of a rewriting rule from $\mathcal{R}$ always decreases a word in the lexicographic order, it follows that no rule in $\mathcal{R}$ can be applied to $w$. Thus $w$ is irreducible.

Now we prove that any irreducible word arises as a left-to-rise postfix reading of a binary search tree. We proceed by induction on the length of the word. First, if $w \in A^{*}$ has length less than 3 , no relation in $\mathcal{R}$ can be applied to $w$ and hence there is no other word in $A^{*}$ representing the same element of $S_{n}$; thus $w=\operatorname{LRP}(\mathcal{T}(w))$. This proves the base of the induction.

Now suppose that all irreducible words of length less than $k$ arise from left-to-right postfix readings of binary search trees. Let $w$ be an irreducible word of length $k$. Let $w=w^{\prime} b$, where $b \in A$. Since $w$ is irreducible, there is no left-hand side of a rule in $\mathcal{R}$ in $w$, and thus there is no subword ca in $w^{\prime}$ such that $\mathrm{a} \leqslant \mathrm{b}<\mathrm{c}$. Therefore $w^{\prime}$ factors as $w^{\prime}=\mathfrak{u} v$, where every symbol in $u$ is less than or equal to $b$ and every symbol in $v$ is greater than $b$. The words $u$ and $v$ are irreducible and of length less than $k$, and thus $u=\operatorname{LRP}\left(T_{u}\right)$ and $v=\operatorname{LRP}\left(\mathrm{T}_{v}\right)$ for some binary search trees $\mathrm{T}_{u}$ and $\mathrm{T}_{v}$. Form a new tree $T$ whose root is labelled by $b$ and has left subtree $T_{u}$ and right subtree $T_{v}$. Since every symbol in $u$ is less or equal to than $b$ and every symbol in $v$ is greater than $v$, it follows that $\mathrm{T}$ is a binary search tree. Finally, by the definition of left-to-right postfix reading, we have $\operatorname{LRP}(T)=\operatorname{LRP}\left(T_{u}\right) \operatorname{LRP}\left(T_{v}\right) b=u v b=w$. This completes the induction step.

Thus irreducible words are precisely those arising from left-to-right postfix readings of binary search trees, which are precisely the lexicographically minimal representatives of the elements of $S_{n}$ by Proposition 5.2. Hence there is a unique irreducible representative of each element of $S_{n}$, and so $(A, \mathcal{R})$ is confluent.

As a consequence of Proposition 5.3, $S_{n}$ admits a regular language of unique normal forms, namely the language of words that do not include a left-hand side of a rule in $\mathcal{R}$ :

$$
L=A^{*}-A^{*}\left\{c a v b: a \leqslant b<c, v \in A^{*}\right\} A^{*} .
$$

Thus $L$ is the language of irreducible words of the rewriting system $(A, \mathcal{R})$.

\subsection{Biautomaticity}

The aim of this section is to prove that $(A, L)$ is a biautomatic structure for $S_{n}$. It is necessary to prove that ${ }_{\gamma} L \delta_{L}, \gamma L \delta_{R}, L_{\gamma} \delta_{L}$, and $L_{\gamma} \delta_{R}$ are regular.

\subsubsection{Left-multiplication}

We begin by analyzing the reduction that can occur when we left-multiply a normal form word by a single generator and then rewrite back 
to a normal form word. Let $w \in \mathrm{L}$ and $\gamma \in A$ and consider reducing $\gamma w$ to normal form.

Lemma 5.4. After $\mathrm{k}$ steps, the reduction of $\gamma w$ must yield a word $\mathfrak{u}_{\mathrm{k}} \gamma v_{\mathrm{k}}$ such that

1. $w=u_{k} v_{k} ;$

2. $\gamma$ is greater than every symbol of $\mathfrak{u}_{k}$;

3. if a rule cavb $\rightarrow_{\mathcal{R}} \mathrm{ac} v \mathrm{~b}$ applies to $\mathrm{u}_{\mathrm{k}} \gamma v_{\mathrm{k}}$, then $\mathrm{c}$ is the distinguished symbol $\gamma$, the first symbol of $v_{\mathrm{k}}$ is $\mathrm{a}$, and some other symbol of $v_{\mathrm{k}}$ is $\mathrm{b}$.

Proof of 5.4. The proof is by induction on $k$. Let $u_{0}=\varepsilon$ and $v_{0}=w$. Then for $k=0$, conditions 1 and 2 hold immediately. Since $v_{k}=w$ is irreducible, if any rule in $\mathcal{R}$ applies to $u_{\mathrm{k}} \gamma v_{\mathrm{k}}=\gamma w$, it must apply as condition 3 specifies. This proves the base case of the induction.

Now assume that the result holds for $k=\ell-1$; we aim to show it holds for $k=\ell$. So after $\ell-1$ steps, reduction of $\gamma w$ yields $u_{\ell-1} \gamma v_{\ell-1}$ with conditions $1-$ 3 being satisfied for $k=\ell-1$. We will use the fact that conditions $1-3$ hold for $k=\ell-1$ to prove conditions 1 and 2 for $k=\ell$; condition 3 for $k=\ell$ then follows from conditions 1 and 2 for $k=\ell$.

If $u_{\ell-1} \gamma v_{\ell-1}$ is irreducible, no further reduction takes place and there is nothing more to prove. So suppose $u_{\ell-1} \gamma v_{\ell-1}$ is not irreducible. Then by condition 3 for $k=\ell-1$ we can let $\alpha \in A$ and $\nu_{\ell} \in A^{*}$ be such that $\nu_{\ell-1}=\alpha v_{\ell}$ and any rule in $\mathcal{R}$ that applies to $u_{\ell-1} \gamma v_{\ell-1}$ has $c=\gamma, a=\alpha$, and $b$ being some symbol in $v_{\ell}$. Note that by the definition of $\mathcal{R}$, we have $c>a$ and so $\gamma>\alpha$. Applying this rule yields $\mathfrak{u}_{\ell-1} \alpha \gamma v_{\ell}$. Let $u_{\ell}=u_{\ell-1} \alpha$.

By condition 1 for $k=\ell-1$, we have $w=u_{\ell-1} v_{\ell-1}$ and so $w=u_{\ell-1} \alpha v_{\ell}=$ $u_{\ell} v_{\ell}$; this establishes condition 1 for $k=\ell$.

By condition 2 for $k=\ell-1$, we know $\gamma$ is greater than every symbol of $\mathfrak{u}_{\ell-1}$. Since $\gamma>\alpha$, we deduce that $\gamma$ is greater than every symbol of $\mathfrak{u}_{\ell}$; this proves condition 2 for $k=\ell$.

Finally, suppose some rule cavb $\rightarrow_{\mathcal{R}} a c v b$ (where $a \leqslant b<c$ ) applies to $\mathfrak{u}_{\ell} \gamma v_{\ell}$. If $b$ lies in $\mathfrak{u}_{\ell}$, then cavb is a subword $\mathfrak{u}_{\ell}$ and thus of $w$, which contradicts $w$ being irreducible. If $\mathrm{b}$ is the distinguished letter $\gamma$, then $\mathrm{c}$ lies in $u_{\ell}$, which contradicts condition 2 for $k=\ell$. Hence $b$ must be some symbol of $v_{\ell}$. If $c a$ is a subword of either $u_{\ell}$ or $v_{\ell}$, then $c a v^{\prime} b$ is a subword of $w=u_{\ell} v_{\ell}$ for some $v^{\prime}$, contradicting the irreducibility of $w$. If $a$ is the distinguished letter $\gamma$, then $c$ lies in $u_{k}$, again contradicting the condition 2 for $k=\ell$. The only remaining possibility is that $\mathrm{c}$ is the distinguished symbol $\gamma$, with a being the first symbol of $v_{k}$. This proves condition 3 for $k=\ell$.

Let $w \in \mathrm{L}$ and $\gamma \in A$. By Lemma 5.4, rewriting $\gamma w$ to an irreducible word consists of moving $\gamma$ to the right by applying rules cavb $\rightarrow_{\mathcal{R}}$ ac $v b$. Let us consider the symbols in $\gamma w$ that play the role of $b$ in these rules. Let $\beta_{1}$ be the first symbol playing this role. Apply rules involving $\beta_{1}$ as many times as possibly, say $k_{1}$ times. This yields a word $u_{k_{1}} \gamma v_{k_{1}}$. Let $\alpha_{k_{1}}$ be the first symbol of $v_{k_{1}}$. Suppose this word $u_{k_{1}} \gamma v_{k_{1}}$ is not irreducible. So some rule from $\mathcal{R}$ applies, with some symbol $\beta_{2} \neq \beta_{1}$ playing the role of $b$. Then $\alpha_{k_{1}}>\beta_{1}$ (since otherwise a rule from $\mathcal{R}$ would apply with $c=\gamma, a=\alpha_{k_{1}}$, and $b=\beta_{1}$ ). Since a rule applies with $c=\gamma, a=\alpha_{k_{1}}$, and $b=\beta_{2}$, we have $\beta_{2} \geqslant \alpha_{k_{1}}>\beta_{1}$. Apply rules involving $\beta_{2}$ as many times as possible, say $k_{2}$ times, yielding $u_{k_{2}} \gamma v_{k_{2}}$. Either this word is irreducible or, via the reasoning above, it can be reduced by a rule in $\mathcal{R}$ with $b$ being $\beta_{3}>\beta_{2}$. Repeating this reasoning, 
we set a sequence $\beta_{1}<\beta_{2}<\ldots$ which must terminate at some $\beta_{\ell}$ with an irreducible word $u_{k_{\ell}} \gamma v_{k_{\ell}}$ since the alphabet $A$ is finite.

Let

$$
\begin{aligned}
H_{b}=\{(\text { pcqrbs, pqcrbs }): & p, r, s \in A^{*}, \\
& b, c \in A, \\
& c>b, \\
& \left.q \in\{a \in A: a \leqslant b\}^{+}\right\} .
\end{aligned}
$$

Clearly, (pcqrbs, pqcrbs) $\in \mathrm{H}_{\mathrm{b}}$ if and only if pcqrbs reduces to pqcrbs using only rules from $\mathcal{R}$ applied to the distinguished letters $c$ and $b$ with $a$ being the successive letters of $\mathrm{q}$. Hence, by the reasoning in the preceding paragraph, and using the same notation,

$$
\left(\gamma w, u_{k_{\ell}} \gamma v_{k_{\ell}}\right) \in \mathrm{H}_{\beta_{1}} \circ \mathrm{H}_{\beta_{2}} \circ \cdots \circ \mathrm{H}_{\beta_{\ell}} .
$$

Therefore,

$$
(w, x) \in_{\gamma} \mathrm{L} \Longleftrightarrow(\gamma w, x) \in((\gamma \mathrm{L}) \times \mathrm{L}) \cap \bigcup_{\ell=0}^{n} \bigcup_{\substack{\beta_{1}, \ldots, \beta_{\ell} \in A \\ \beta_{1}<\ldots<\beta_{\ell}}} \mathrm{H}_{\beta_{1}} \circ \cdots \circ \mathrm{H}_{\beta_{\ell}}
$$

or equivalently

$$
(\gamma, \varepsilon)_{\gamma} \mathrm{L}=((\gamma \mathrm{L}) \times \mathrm{L}) \cap \bigcup_{\ell=0}^{\mathrm{n}} \bigcup_{\substack{\beta_{1}, \ldots, \beta_{\ell} \in A \\ \beta_{1}<\ldots<\beta_{\ell}}} \mathrm{H}_{\beta_{1}} \circ \cdots \circ \mathrm{H}_{\beta_{\ell}} .
$$

(Note that the intersection with $(\gamma \mathrm{L}) \times \mathrm{L}$ is necessary because $\mathrm{H}_{\mathrm{b}}$ also relates pairs of words that are not in this set.)

It is easy to see that $\mathrm{H}_{\mathrm{b}}$ is a rational relation, since a transducer recognizing it only needs to store the symbol $\mathrm{c}$ in its state, check that the other symbols on the two tapes match, and that the contents of the two tapes are of the required form. Hence $(\gamma, \varepsilon)_{\gamma} \mathrm{L}$ is a rational relation and so $\gamma \mathrm{L}$ is a rational relation. Since $(w, x) \in{ }_{\gamma} L$ implies $|x|=|w|+1$, it follows from Proposition 2.3 that ${ }_{\gamma} \mathrm{L} \delta_{\mathrm{R}}$ and ${ }_{\gamma} \mathrm{L} \delta_{\mathrm{L}}$ are regular.

\subsubsection{Right-multiplication}

We now turn to right multiplication. By analogy with the hypoplactic monoid, we will call any strictly decreasing word in $A^{*}$ a column. Notice that since $A$ is finite, there are only finitely many distinct columns.

Lemma 5.5. Let $w \in \mathrm{L}$, and let $w=\alpha^{(1)} \cdots \alpha^{(\mathrm{k})}$ be the decomposition of $w$ into maximal columns. If $\alpha^{(i)}=\alpha^{(i+h)}$ with $h \geqslant 1$, then $\left|\alpha^{(i)}\right|=\left|\alpha^{(i+h)}\right|=1$.

Proof of 5.5. Suppose that $\alpha^{(i)}=\alpha^{(i+h)}$. Suppose, with the aim of obtaining a contradiction, that $\left|\alpha^{(i)}\right| \geqslant 2$. Suppose $\alpha^{(i)}=\alpha^{\prime} c a$, where $c, a \in A$ and $\alpha^{\prime} \in A^{*}$. Let $b=a$. Then $c>a$ (since $\alpha^{(i)}$ is a decreasing word) and so the condition $\mathrm{a} \leqslant \mathrm{b}<\mathrm{c}$ holds. Thus the rewriting rule $\mathrm{cavb} \rightarrow_{\mathcal{R}} \mathrm{ac} v \mathrm{~b}$ applies with $c$, $a$ being the rightmost two symbols in $\alpha^{(i)}$ and $b$ being the rightmost symbol in $\alpha^{(i+h)}$ (which is, by hypothesis, equal to $\alpha^{(i)}$ ). Hence $w$ is not irreducible, which contradicts $w$ lying in L 
We will analyze the reduction that can occur when we right-multiply a normal form word by a single generator and then rewrite back to a normal form word. Let $w \in \mathrm{L}$ and $\gamma \in A$ and consider reducing $w \gamma$ to normal form. Suppose $w=\alpha_{1} \cdots \alpha_{|w|}$ for $\alpha_{i} \in A$. Let $G=\left\{i \in\{1, \ldots,|w|\}: \alpha_{i} \leqslant \gamma\right\}$ and consider the word

$$
x=\left[\prod_{\substack{1 \leqslant i \leqslant|w| \\ i \in G}} \alpha_{i}\right]\left[\prod_{\substack{1 \leqslant j \leqslant|w| \\ j \notin G}} \alpha_{j}\right] \gamma .
$$

LemMA 5.6. The word $x$ is irreducible with respect to $\mathcal{R}$, and $w \gamma \rightarrow_{\mathcal{R}}^{*} x$. Furthermore, rewriting of $w \gamma$ to $x$ only requires applying rules from $\mathcal{R}$ with $\mathrm{b}=\gamma$.

Proof of 5.6. First of all, notice that the rules in $\mathcal{R}$ apply with $b=\gamma$ to move all letters less than or equal to $\gamma$ to the left of those strictly greater than $\gamma$. So $w \gamma$ certainly rewrites to $x$ in the given way; it remains to show that $x$ is irreducible.

Suppose, with the aim of obtaining a contradiction, that some rule in $\mathcal{R}$ applies to $x$. Then $x$ contains a subword cavb for some $a \leqslant b<c$ and $v \in A^{*}$.

Now, it is impossible to have $b=\gamma$, for this implies $a=\alpha_{i}$ for some $i \in G$ and $c=\alpha_{j}$ for some $j \notin G$, which in turn implies that $a$ appears to the left of $c$ in $x$, which contradicts the form of the subword cavb.

It is also impossible to have $c=\alpha_{i}$ for $i \in G$ and $b=\alpha_{j}$ for $j \notin G$, for then (by definition of G) $c \leqslant \gamma<b$, contradicting the form of the subword cavb.

So $c$ and $b$ (and hence the whole subword $c a v b$ ) must either both lie within the product of the $\alpha_{i}$ with $i \in G$, or both lie within the product of the $\alpha_{j}$ with $j \notin G$. In either case, if the letters ca were adjacent in the original word $w$, it would not have been irreducible, since the rewriting of $w \gamma$ to $x$ using $\mathcal{R}$ preserves the order in which the $\alpha_{i}$ (with $i \in G$ ) appear and the order in which the $\alpha_{j}$ (with $j \notin \mathrm{G}$ ) appear.

Consider first the case that cavb lies wholly within the product $\alpha_{i}$ with $i \in G$. Then, since ca were not adjacent in the original word $w$, we have $c=\alpha_{i}$ and $a=\alpha_{i+h}$ with $i, i+h \in G$ and $i+1, \ldots, i+h-1 \notin G$ for some $h \geqslant 2$. Let $c^{\prime}=\alpha_{i+h-1}$. Then, by definition of $G$, we have $a \leqslant b<c^{\prime}$ and so there is a left-hand side of a rule in $\mathcal{R}$ in $w$, contradicting $w$ being irreducible.

Now consider the second case, where cavb lies wholly within the product $\alpha_{j}$ with $j \notin G$. Then, since ca were not adjacent in the original word $w$, we have $c=\alpha_{j}$ and $a=\alpha_{j+h}$ with $j, j+h \notin G$ and $j+1, \ldots, j+h-1 \in G$ for some $h \geqslant 2$. Let $a^{\prime}=\alpha_{j+1}$. Then, by definition of $G$, we have $a^{\prime} \leqslant b<c$ and so there is a left-hand side of a rule in $\mathcal{R}$ in $w$, contradicting $w$ being irreducible.

So each case leads to a contradiction. Hence $x$ is irreducible.

Recall that a word $w \in \mathrm{L}$ admits a decomposition $w=\alpha^{(1)} \alpha^{(2)} \ldots \alpha^{(\mathrm{k})}$ into maximal columns. Let I be the set of indices $i$ such that the letter $\alpha_{i}$ of $w$ is itself one of the columns $\alpha^{(j)}$. That is, $\alpha_{i}$ does not lie in a column $\alpha^{(j)}$ containing two or more symbols from $A$. Since the length of columns is bounded by $n$, there is a bounded number of possible columns of length at least 2. Each of these columns appears at most once in $w$ by Lemma 5.5. Since each of these columns has length at most $n$, there is a bound $M$ (dependent only on $n$ ) on the number of indices not in I.

LEMMA 5.7. For all $i, i+h \in I$, we have $\alpha_{i} \leqslant \alpha_{i+h}$.

Proof of 5.7. Suppose, with the aim of obtaining a contradiction, that for some $i, i+h \in I$, we have $\alpha_{i}>\alpha_{i+h}$. Since $\alpha_{i+h}$ is a column (a maximal strictly 
decreasing subword of), we have $\alpha_{i+h-1} \leqslant \alpha_{i+h}$. In particular, $h \geqslant 2$. Since $\alpha_{i}>\alpha_{i+h} \geqslant \alpha_{i+h-1}$, the sequence $\alpha_{i}, \ldots, \alpha_{i+h-1}$ starts greater than $\alpha_{i+h}$ and ends less than or equal to $\alpha_{i+h}$. So there must be some $g \in\{0, \ldots, h-2\}$ such that $\alpha_{i+g}>\alpha_{i+h} \geqslant \alpha_{i+g+1}$. Hence a rule from $\mathcal{R}$ applies to $w$ with $\mathrm{c}=$ $\alpha_{i+g}, a=\alpha_{i+g+1}$, and $b=\alpha_{i+h}$, which contradicts $w$ being irreducible. 5.7

Thus, the rewriting of $w \gamma$ to $x$ does not alter the relative positions of symbols $\alpha_{i}$ with $i \in$ I. So only symbols with subscripts not in I have to be moved rightwards using rules in $\mathcal{R}$ with $b=\gamma$ in order to rewrite $w \gamma$ to $x$. That is, at most $M$ symbols, each greater than $\gamma$, must be moved to the right of all symbols less or equal to $\gamma$ (excepting $\gamma$ itself).

Since the symbol $\gamma$ is not moved during this rewriting, we will consider the relation

$$
\mathrm{L}_{\gamma}^{\prime}=\left\{\left(w, x^{\prime}\right): w \in \mathrm{L}, w \rightarrow \rightarrow_{\mathcal{R}}^{*} x^{\prime} \gamma \in \mathrm{L}\right\},
$$

Notice that $\mathrm{L}_{\gamma}=\mathrm{L}_{\gamma}^{\prime}(\varepsilon, \gamma)$, and so if a synchronous transducer recognizes $\mathrm{L}_{\gamma}^{\prime}$, it recognizes $\mathrm{L}_{\gamma}$.

Let

$$
\begin{aligned}
J_{\gamma}=\{(\text { pcqr, pqcr }): & p \in A^{*} \\
& q \in\{a \in A: a \leqslant \gamma\}^{+} \\
& r \in\{a \in A: a>\gamma\}^{*} \\
& c \in A, c>\gamma\} .
\end{aligned}
$$

Notice that if (pcqr,pqcr) $\in \mathrm{J}_{\gamma}$, then pqcr $\gamma$ is the word obtained from pcqr $\gamma$ by applying rewriting rules $c a v b \rightarrow_{\mathcal{R}} a c v b$ with $\gamma$ being $b$ and successive letters from $q$ being $a$. Thus if we start with our word $w$ and apply $J_{\gamma}$, we move the rightmost letter $c$ that is greater than $\gamma$ but which lies to the left of some letter less than or equal to $\gamma$ into its proper place. Iterating this process will therefore yield $x^{\prime}$. Since there are at most $M$ symbols that have to be moved rightwards to their proper places to obtain $x^{\prime}$ from $w$, at most $M$ iterations are required. Therefore

$$
\mathrm{L}_{\gamma}^{\prime}=(\mathrm{L} \times(\mathrm{L} / \gamma)) \cap \bigcup_{\ell=1}^{\mathrm{M}} \underbrace{\mathrm{J}_{\gamma} \circ \cdots \circ \mathrm{J}_{\gamma}}_{\ell \text { times }}
$$

(Recall that $\mathrm{L} / \gamma=\left\{w \in A^{*}: w \gamma \in \mathrm{L}\right\}$. Note that we have to take the intersection with $\mathrm{L} \times(\mathrm{L} / \gamma)$ because $\mathrm{J}_{\gamma} \circ \cdots \circ \mathrm{J}_{\gamma}$ may contains pairs $(\mathrm{y}, z)$ where $\mathrm{y}$ and $z \gamma$ are not irreducible.)

It is easy to see that $J_{\gamma}$ is a rational relation, since a transducer recognizing it only needs to store the symbol $\mathrm{c}$ in its state, check that the other symbols on the two tapes match, and that the contents of the two tapes are of the required form. Hence $L_{\gamma}^{\prime}$ is a rational relation and so $L_{\gamma}$ is a rational relation. Since $(w, x) \in \mathrm{L}_{\gamma}$ implies $|x|=|w|+1$, it follows from Proposition 2.3 that $\mathrm{L}_{\gamma} \delta_{\mathrm{R}}$ and $\mathrm{L}_{\gamma} \delta_{\mathrm{L}}$ are regular.

In the previous section, we proved that ${ }_{\gamma} L \delta_{R}$ and ${ }_{\gamma} L \delta_{L}$ are regular. Thus we have proved:

THEOREM 5.8. (A, L) is a biautomatic structure for the sylvester monoid $\mathrm{S}_{\mathrm{n}}$. 


\section{REFERENCES}

[Ber79] J. Berstel. Transductions and context-free languages, vol. 38 of Leitfäden der Angewandten Mathematik und Mechanik [Guides to Applied Mathematics and Mechanics]. B.G. Teubner, Stuttgart, 1979.

[BN98] F. Baader $\mathcal{E}$ T. Nipkow. Term rewriting and all that. Cambridge University Press, Cambridge, 1998.

$\left[\mathrm{CEK}^{+}\right.$o1] J. Cassaigne, M. Espie, D. Krob, J. C. Novelli, \& F. Hivert. 'The Chinese monoid'. Internat. J. Algebra Comput., 11, no. 3 (2001), pp. 301-334. DoI: 10.1142/S0218196701000425.

[CGM] A. J. Cain, R. Gray, \& A. Malheiro. 'On finite complete rewriting systems, finite derivation type, and automaticity for homogeneous monoids'. arXiv: 1407.7428.

[CGM15] A. J. Cain, R. D. Gray, \& A. Malheiro. 'Finite Gröbner-Shirshov bases for Plactic algebras and biautomatic structures for Plactic monoids'. J. Algebra, 423 (2015), pp. 37-53. arXiv: 1205.4885, DOI: 10.1016/j.jalgebra.2014.09.037.

[CM] A. J. Cain \& A. Malheiro. 'Deciding conjugacy in sylvester monoids and other homogeneous monoids'. arXiv: 1404.2618.

[CO13] F. Cedó \& J. Okniński. 'Minimal Spectrum and the Radical of Chinese Algebras'. Algebr. Represent. Theory, 16, no. 4 (2013), pp. 905-930. Dor: 10.1007/s10468-012-9339-1.

[CQo8] Y. Chen \& J. Qiu. 'Gröbner-Shirshov basis for the Chinese monoid'. J. Algebra Appl., 7, no. 5 (2008), pp. 623-628. DOI: 10.1142/So219498808003028.

[CRRTo1] C. M. Campbell, E. F. Robertson, N. Ruškuc, E R. M. Thomas. 'Automatic semigroups'. Theoret. Comput. Sci., 250, no. 1-2 (2001), pp. 365-391. DOI: 10.1016/S0304-3975(99)00151-6.

[DK94] G. Duchamp \& D. Krob. 'Plactic-growth-like monoids'. In Words, languages and combinatorics, II (Kyoto, 1992), pp. 124-142. World Sci. Publ., River Edge, NJ, 1994.

[DRR99] A. J. Duncan, E. F. Robertson, $\mathcal{E}$ N. Ruškuc. 'Automatic monoids and change of generators'. Math. Proc. Cambridge Philos. Soc., 127, no. 3 (1999), pp. 403-409. DoI: 10.1017/S0305004199003722.

$\left[\mathrm{ECH}^{+}{ }^{92}\right]$ D. B. A. Epstein, J. W. Cannon, D. F. Holt, S. V. F. Levy, M. S. Paterson, $\mathcal{E}$ W. P. Thurston. Word Processing in Groups. Jones \& Bartlett, Boston, Mass., 1992.

[FS93] C. Frougny $\mathcal{E}$ J. Sakarovitch. 'Synchronized rational relations of finite and infinite words'. Theoret. Comput. Sci., 108, no. I (1993), pp. 4582. International Colloquium on Words, Languages and Combinatorics (Kyoto, 1990). DOI: 10.1016/0304-3975(93)90230-Q.

[GK1o] E. Güzel Karpuz. 'Complete rewriting system for the Chinese monoid'. Appl. Math. Sci. (Ruse), 4, no. 21-24 (2010), pp. 1081-1087.

[Heyoo] A. Heyworth. 'Rewriting as a special case of non-commutative Gröbner basis theory'. In Computational and geometric aspects of modern algebra (Edinburgh, 1998), vol. 275 of London Math. Soc. Lecture Note Ser., pp. 101-105. Cambridge Univ. Press, Cambridge, 2000. DOI: 10.1017/CBO9780511600609.009.

[HNTo5] F. Hivert, J. C. Novelli, \& J. Y. Thibon. 'The algebra of binary search trees'. Theoret. Comput. Sci., 339, no. 1 (2005), pp. 129-165. DoI: 10.1016/j.tcs.2005.01.012.

[HTo5] M. Hoffmann $\mathcal{E}$ R. M. Thomas. 'Biautomatic semigroups'. In Fundamentals of computation theory, vol. 3623 of Lecture Notes in Comput. Sci., pp. 56-67. Springer, Berlin, 2005. DoI: 10.1007/11537311_6. 
[HU79] J. E. Hopcroft $\mathcal{E}$ J. D. Ullman. Introduction to Automata Theory, Languages, and Computation. Addison-Wesley Publishing Co., Reading, Mass., 1979.

[JOo6] J. Jaszuńska \& J. Okniński. 'Chinese algebras of rank 3'. Comm. Algebra, 34, no. 8 (2006), pp. 2745-2754. DoI: 10.1080/00927870600651760.

[JO11] J. Jaszuńska \& J. Okniński. 'Structure of Chinese algebras'. J. Algebra, 346 (2011), pp. 31-81. DoI: 10.1016/j.jalgebra.2011.08.020.

[KT97] D. Krob \& J. Y. Thibon. 'Noncommutative symmetric functions. IV. Quantum linear groups and Hecke algebras at $q=0^{\prime}$. J. Algebraic Combin., 6, no. 4 (1997), pp. 339-376. DoI: 10.1023/A:1008673127310.

[Loto2] M. Lothaire. Algebraic combinatorics on words, vol. 90 of Encyclopedia of Mathematics and its Applications. Cambridge University Press, Cambridge, 2002.

[LR98] J. L. Loday $\mathcal{E}$ M. O. Ronco. 'Hopf algebra of the planar binary trees'. Adv. Math., 139, no. 2 (1998), pp. 293-309. DoI: 10.1006/aima.1998.1759.

[Novoo] J. C. Novelli. 'On the hypoplactic monoid'. Discrete Math., 217, no. 1-3 (2000), pp. 315-336. Formal power series and algebraic combinatorics (Vienna, 1997). DoI: 10.1016/So012-365X(99)00270-8.

[Ott84] F. Otto. 'Conjugacy in monoids with a special Church-Rosser presentation is decidable'. Semigroup Forum, 29, no. 1-2 (1984), pp. 223-240. DOI: $10.1007 / \mathrm{BF} 02573327$.

[Ufn95] V. A. Ufnarovskij. 'Combinatorial and asymptotic methods in algebra'. In Algebra, VI, vol. 57 of Encyclopaedia of Mathematical Sciences, pp. 1196. Springer, Berlin, 1995. 